\title{
Mesh Objective Tensile Cracking Via a Local Continuum Damage Model and a Crack Tracking Technique
}

M. Cervera M. Chiumenti 


\section{Mesh Objective Tensile Cracking Via a Local Continuum Damage Model and a Crack Tracking Technique}

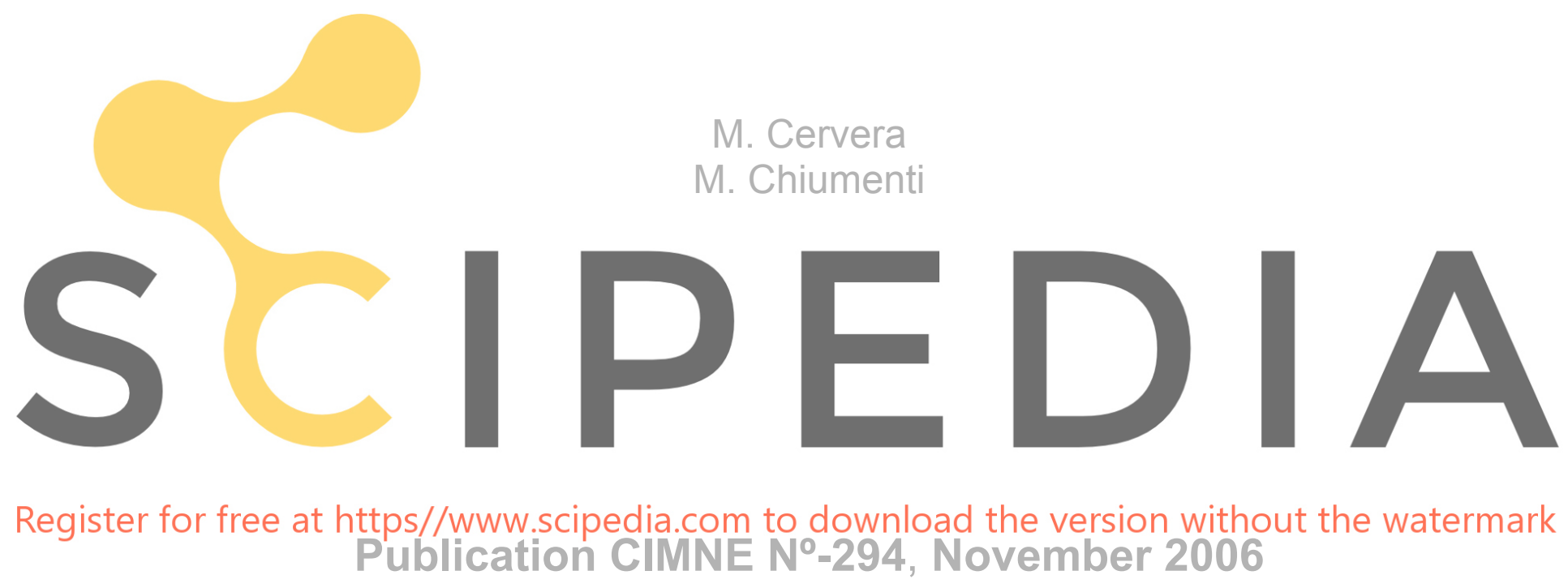




\section{Mesh objective tensile cracking via a local continuum damage model and a crack tracking technique}

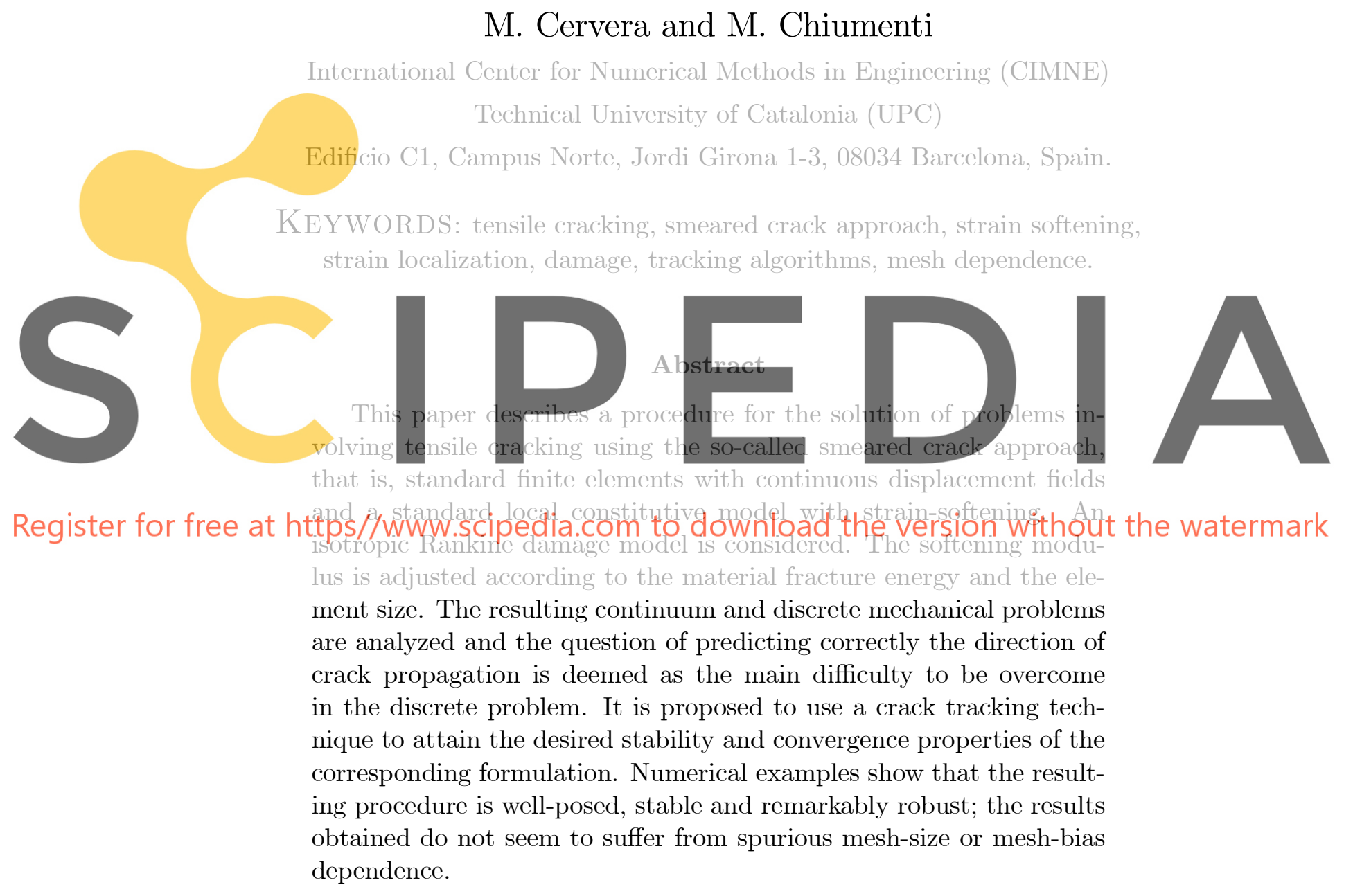




\section{Introduction and Motivation}

Structural failure due to catastrophic crack propagation in some building materials poses problems of design and analysis in many fields of engineering. Geomaterials such as concrete and rocks fail mainly due to tensile straining, and codes of practice in civil engineering address this feature extensively. In aerospace engineering, where safety and over-design must be counterbalanced, the subject of tensile (and fatigue) cracking is of paramount importance. Tensile cracking is also of primary concern in advanced composite materials, and in specific brittle materials like ceramics, glass and ice.

It was early discovered that cracks are present to some degree in all structures. They may be present as basic defects in the constituent materials, or they may be induced by inadequate design or construction or during service life. Therefore, it was very soon realized that means for assessing the stability of such cracks were necessary.

For instance, Galileo Galilei [1], in the XVII century, observed that big ships were proner to tensile cracking than smaller ships, because they were

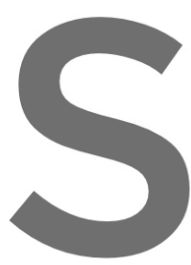
more brittle introduced the first his investigatio unstable when existing crack In 1959
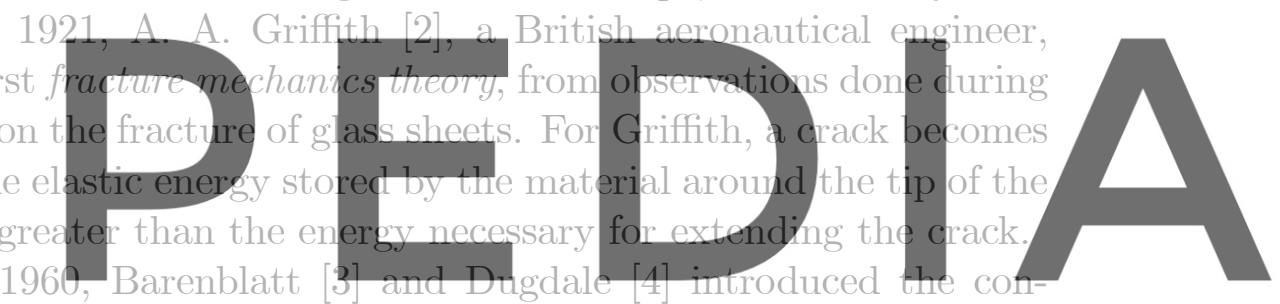

cept of cohesive forces in the crack tip region, the first within the limits of

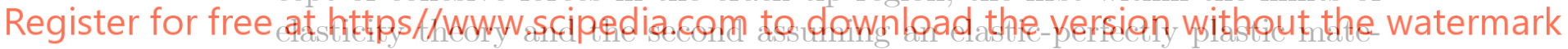

rial behaviour. These were the first attempts to bring closer the theories of fracture mechanics (FM) and continuum mechanics (CMi).

About the same time, the Finite Element Method (FEM) and digital computers dashed into the engineering community as a gifted means for quantifying solutions in structural and solid mechanics. Naturally, fracture mechanicians implemented their FE methods, while continuum mechanicians implemented theirs. This led to the consolidation of two different concepts of the phenomenon of cracking: the discrete and the smeared crack approaches.

The discrete crack (DC) approach is usually based in the FM theory. This means that the criteria for crack propagation and, eventually, the prediction of the direction of propagation come directly from this theory, which is, mostly, based on energy criteria. DC models conceive the individual cracks as actual discontinuities in the topology of the FE mesh. 


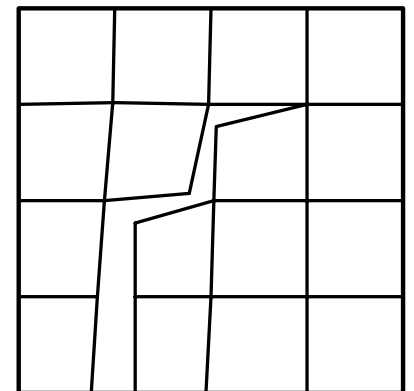

(a)
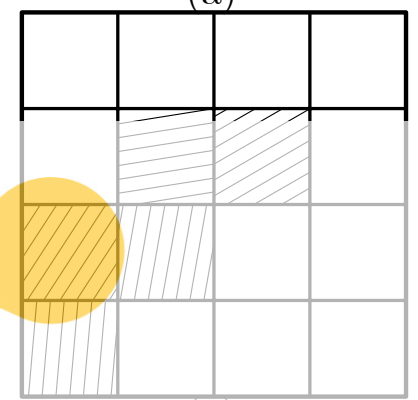

(d)

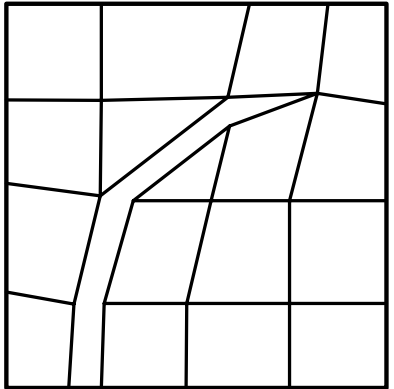

(b)

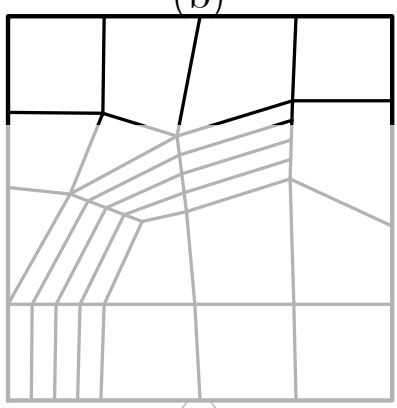

(e)

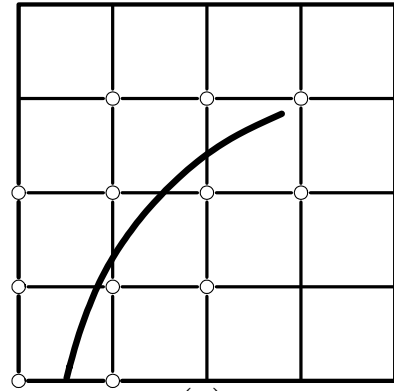

(c)

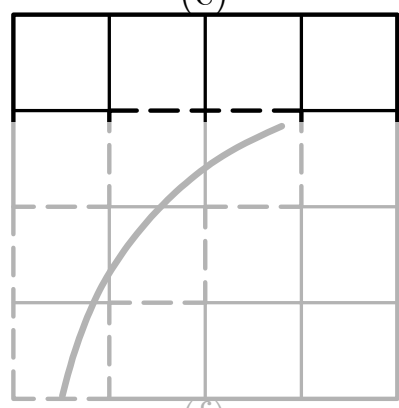

(f)
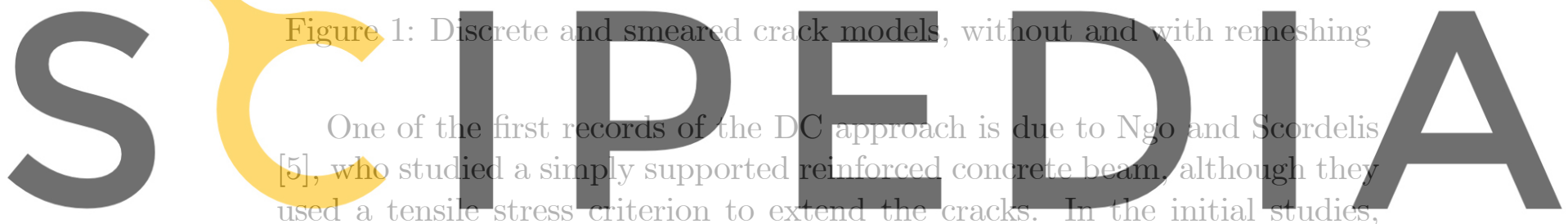

cracks were modelled by separation of nodal points initially occupying the

Register for free at

as cracks could only form along the element boundaries (Fig. 1a). The DC

approach was later refined so that new elements could be introduced whose boundaries were along the spreading crack (Fig. 1b). This reduces the mesh dependency of the approach, but then remeshing techniques are required and the computing time increases. Also, it was recognized almost from the beginning that standard FE were not appropriate to capture the singular stress and strain fields that develop at the tip of the crack [6]; consequently, special FE were developed (see reference [7]).

Recently, Belytschko and coworkers ([8], [9], [10]) have introduced the concept of the extended finite element method (X-FEM). This approach allows for crack propagation without remeshing, at the expense of tracking the advance of the crack through the FE mesh and progressively enriching the nodal degrees of freedom with new ones that represent both the displacement 
jumps across the crack and the developed singular field at the tip of the advancing crack (Fig. 1c, where the "enriched" nodes are marked). Refined integration methods are required for the elements crossed by the crack. With this and other recent developments (see [11] for a review) the need to develop rather complex software, in which the FE model is tightly coupled with the geometrical modeler, has been rather satisfactorily overcome.

On the other hand, the smeared crack (SC) approach is always based in the CM theory, in the sense that the criteria for crack propagation and the prediction of the direction of propagation come directly from this theory, which is, mostly, based on failure criteria expressed in terms of stresses or strains. In $\mathrm{SC}$ models, the cracked material is assumed to remain a continuum and the mechanical properties (stiffness and strength) are modified to account for the effect of cracking, according to the evolving states of strain and/or stress. Therefore, remeshing is, in principle, unnecessary (Fig. 1d).

This implicit simplicity of the approach, proposed by Rashid in his 1968 historical paper [12], caught the attention of the engineering community immediately and, more than 30 years later, many of today commercial FE codes

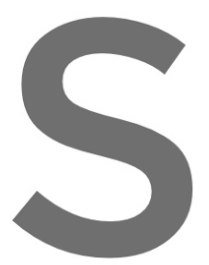
use this appror crack models can be readily implement
writing a routine for a new naterial c
Unfortunately, a drawback of the
was realized in the 1970 's that if a sm the total energy dissipated in the crack
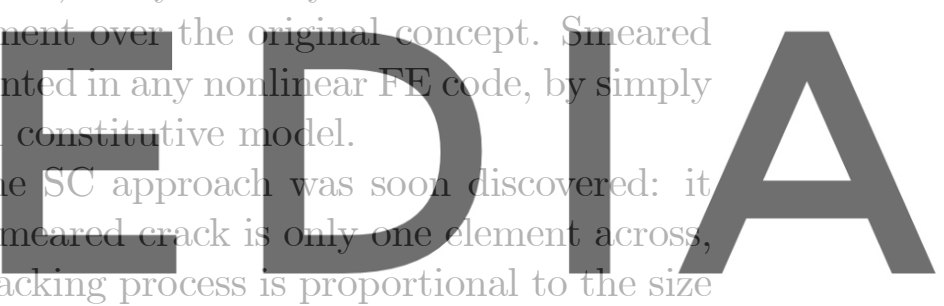
Register for free at (the volume) of the element. Thus, upon mesh refinement, for infinitesimally the physical point of view.

In 1976 Hillerborg et al. [13] formulated, in the context of FM, the "fictitious crack model" (an adaptation of the previously formulated cohesive crack model, already adopted in nonlinear fracture mechanics) and showed that the loss of cohesion in the forming crack should be related to the experimentally measurable fracture energy of the material. In 1983, Bazant and Oh [14] proposed the "crack band model", which is essentially identical to Hillerborg's, but developed in the context of CM and, therefore, easily implemented in standard FE codes. These developments showed that, in the context of FE models, the always controversial concept of strain softening should not be considered as a characteristic of the material, as it is related to the fracture energy of the material and the size of the FE crossed by the smeared crack. This has to be considered as a mile-stone in the road to 
crack modelling because it was the first successful attempt to bring FM and $\mathrm{CM}$ theories to a common standpoint. Today, most of the commercial FE codes implement smeared models with strain softening related to the fracture energy of the material and the element size.

But once the problem of mesh-size dependence was quite satisfactorily overcome, a more difficult one was identifed. In the early 1990's it was widely recognized that FE solutions based on CM suffered from mesh-bias dependence in such a strong manner that it could be ignored. Also, it was noted that if the spatial discretization was designed in such way that an "appropriate" path for the advancing crack was available, the solutions obtained were satisfactory (see Fig. 1e). Again, remeshing was suggested as a partial solution to this problem (see [15], [16]). In the last 15 years, a significant part of the research effort in Computational Solid Mechanics has been devoted to this problem, now termed Computational Failure Mechanics.

To propose, implement and use a computational failure model, set up within the CM framework, three items are necessary: (i) a continuum model that defines the variables and equations of the continuum BVP to be solved,
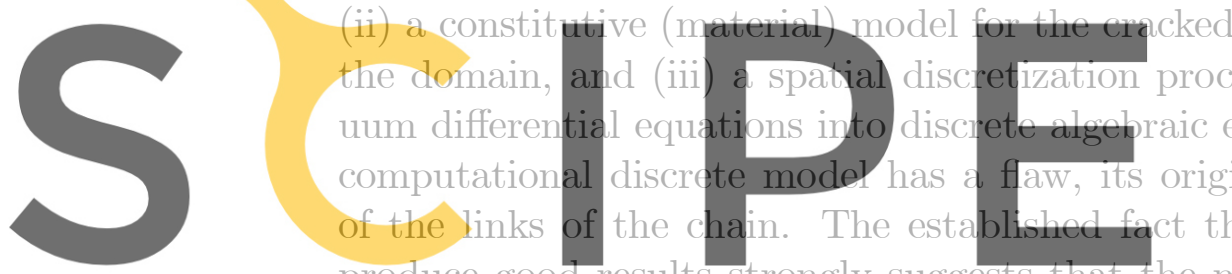
produce good results strongly suggesto

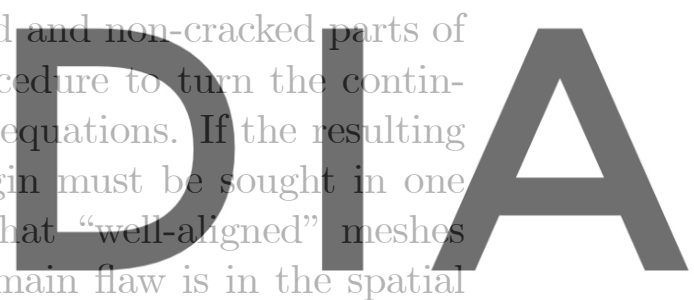
discretization procedure

Register for free at https, WwWW. Scypediancom to downloag the version without the watermark tions have been sought by modifying either the continuum or the constitutive models. In the last decade, many so-called micropolar ([17], [18]), non-local ([17], [19], [20], among others) and gradient-enhanced ([17], [21], [22], [23], [24]) models have been proposed, modifying the standard continuum problem to introduce an internal length that acts as a localization limiter. On one hand, this effectively prevents the development of either strain or displacement discontinuities. On the other hand, even if these strategies have proved effective to some extent, they pose theoretical and computational difficulties, not fully mastered at the present moment. Just to mention a serious one, non-local models do not predict maximum stress values and, therefore, crack initiation, at the tip of a sharp crack, but rather at a finite distance ahead of the tip [25]; this is physically unrealistic. Along a different line, viscousregularized, strain-rate dependent, models (see [17], [24], [26]) do not solve 
the question either, as they also prevent true strain localization and are not effective in the inviscid limit.

Alternatively, the so-called strong discontinuity approach ([27], [28], [29], [30], [31]) represents an effort to tackle the discretization problem directly. The concept of finite elements with embedded discontinuities, as it is also referred to, is certainly appealing, as it does not really depart from the usual continuum framework (its theoretical formulation is very similar to that of contact problems). Interestingly enough, their application invariably needs the use of discontinuity tracking algorithms ([30], [31], [32], [33]), in order to establish which elements lie in the crack and need to be enriched (Fig. 1f, where the elements with embedded discontinuities are marked). This, as the explicit control on the energy dissipated in the formation of the crack, represents another link with the established tradition of fracture mechanics.

Also, in references ([34] and [35]) it is shown that mesh objective solutions, convergent upon refinement and exhibiting highly localized shear bands (or slip lines), can be obtained using standard elements and local $\mathrm{J}_{2}$-plasticity and damage models. The key to obtain these satisfactory solutions is to use

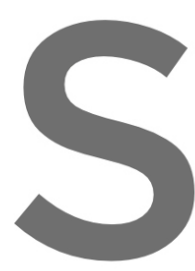
(i) the mixed format of the balance qutations (whic
continuity equation) and (ii) a stabilization techn
fields of the primary variables (displacements and
We attenpt to show in this paper that the
crack propagation problems are related neither to dard continutum equations nor to the

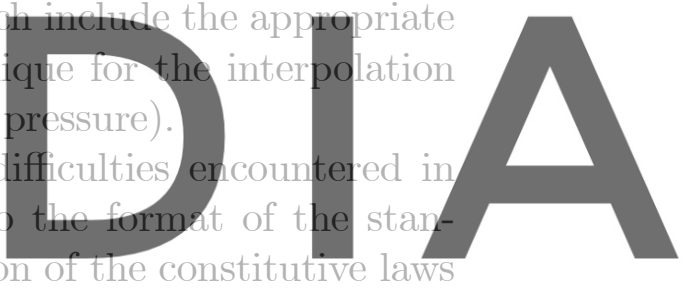
Register for free at hetps//wyw.scipedia com to download the version without the watermark bias encountered in discretized tensile localization problems, (b) to propose a numerical procedure to overcome this, and (c) to assess the performance of the proposed procedure by means of solving selected numerical examples.

The outline of the paper is as follows. In the next section an isotropic scalar Rankine damage model is presented. The necessary adjustment of the softening modulus according to the size of the elements inside the localization band is discussed. Later, the strong and weak forms of the corresponding continuum and discrete problems are presented and the stability of the resulting equations is discussed. Then, the mesh bias dependence observed when using this standard formulation are explained. Tracking of the crack through the FE mesh is presented as a remedy to overcome this problem. Finally, numerical examples are presented to assess the proposed procedure and to show the attained benefits. 


\section{Isotropic Rankine damage model}

\subsection{Constitutive Equation}

The continuum damage mechanics theory is based on the definition of the effective stress, which is introduced in connection with the hypothesis of strain equivalence [36]: the strain associated with a damaged state under the applied stress $\sigma$ is equivalent to the strain associated with its undamaged state under the effective stress $\bar{\sigma}$. In the present work, the effective stresses $\overline{\boldsymbol{\sigma}}$ can be computed in terms of the total strain tensor $\varepsilon, \varepsilon=\nabla^{s} \mathbf{u}$, where $\mathbf{u}$ are the displacements, as

$$
\bar{\sigma}=\mathrm{C}: \varepsilon
$$

where $\mathbf{C}$ is the usual (fourth order) isotropic linear-elastic constitutive tensor, and $(:)$ denotes the double contraction.

The constitutive equation for the damage model is defined as:

$$
\sigma=(1-d) \bar{\sigma}=(1-d) \mathrm{C}: \varepsilon
$$
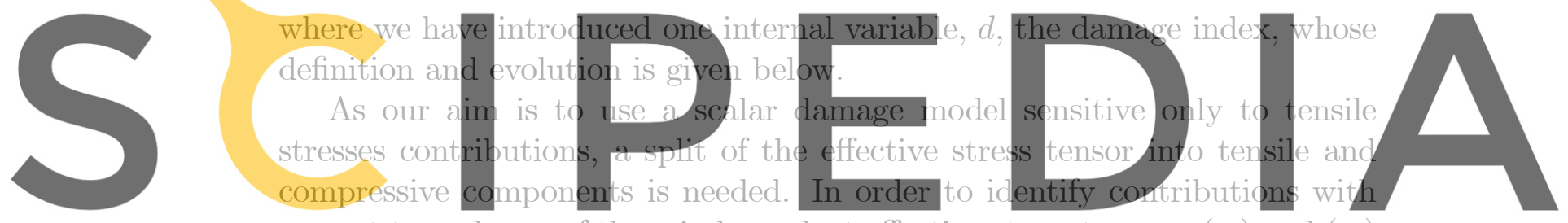

respect to each one of these independent effective stress tensors, $(+)$ and $(-)$

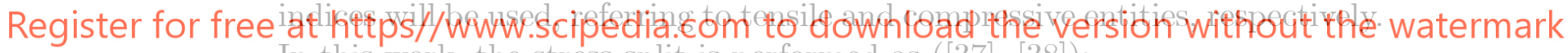

In this work, the stress split is performed as ([37], [38]):

$$
\overline{\boldsymbol{\sigma}}^{+}=\sum_{j=1}^{3}\left\langle\bar{\sigma}_{j}\right\rangle \mathbf{p}_{j} \otimes \mathbf{p}_{j} \quad \text { and } \quad \overline{\boldsymbol{\sigma}}^{-}=\overline{\boldsymbol{\sigma}}-\overline{\boldsymbol{\sigma}}^{+}
$$

where $\bar{\sigma}_{j}$ denotes the $j$-th principal stress value from tensor $\overline{\boldsymbol{\sigma}}, \mathbf{p}_{j}$ represents the unit vector associated with its respective principal direction and the symbol $\otimes$ denotes the tensor product. The symbols $\langle\cdot\rangle$ are the Macaulay brackets $(\langle x\rangle=x$, if $x \geq 0,\langle x\rangle=0$, if $x<0)$.

\subsection{Characterization of Damage}

In order to define concepts such as loading, unloading, or reloading of general $3 \mathrm{D}$ stress states, a scalar positive quantity, termed as equivalent stress, is 
defined. With such a definition, distinct 3D stress states can be mapped to a single equivalent $1 D$ tensile test, which makes their quantitative comparison possible [39], [40].

In the present work, the equivalent stress will assume the following form:

$$
\tau=\left\langle\bar{\sigma}_{1}\right\rangle
$$

where $\bar{\sigma}_{1}$ is the largest principal effective stress. Eq. (4) can written as:

$$
\tau=\left[\overline{\boldsymbol{\sigma}}^{+}: \boldsymbol{\Lambda}: \overline{\boldsymbol{\sigma}}^{+}\right]^{1 / 2}
$$

where the non-dimensional fourth order tensor $\boldsymbol{\Lambda}=\mathbf{p}_{1} \otimes \mathbf{p}_{1} \otimes \mathbf{p}_{1} \otimes \mathbf{p}_{1}$ has been introduced. The role of this tensor is to define the shape of the damage bounding surfaces in a effective stress space, as it will be explained below.

With the above definition for the equivalent effective stress, the damage criterion, $\Phi$, is introduced as:

$$
\Phi(\tau, r)=\tau-r \leq 0
$$
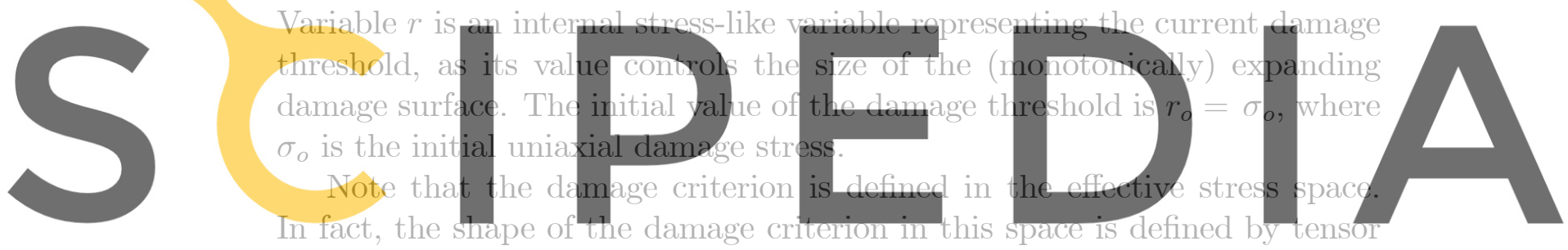

$\Lambda$. As stated before, in this work we will use $\Lambda=\mathrm{p}_{1} \otimes \mathrm{p}_{1} \otimes \mathrm{p}_{1} \otimes \mathrm{p}_{1}$, which

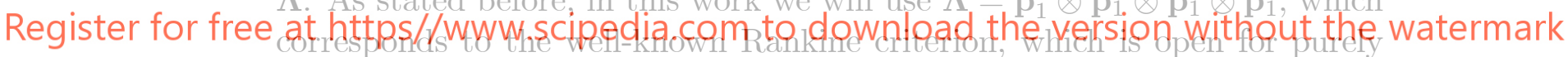
compressive stress states. Figure 2a shows a schematic representation of this damage criterion. An alternative choice $\Lambda=\sum_{j=1}^{3} \mathbf{p}_{j} \otimes \mathbf{p}_{j} \otimes \mathbf{p}_{j} \otimes \mathbf{p}_{j}$ represents a Rankine-type of criterion rounded for biaxial and triaxial tensile states.

The expansion of the damage bounding surface for loading, unloading and reloading conditions is controlled by the Kuhn-Tucker relations and the damage consistency condition, which are

$$
\begin{aligned}
& \dot{r} \geq 0 \quad \Phi(\tau, r) \leq 0 \quad \dot{r} \Phi(\tau, r)=0 \\
& \text { if } \Phi(\tau, r)=0 \text { then } \dot{r} \dot{\Phi}(\tau, r)=0
\end{aligned}
$$

leading, in view of Eq. (6), to the loading condition

$$
\dot{r}=\dot{\tau}
$$


This, in turn, leads to the explicit definition of the current values of the internal variable $r$ in the form

$$
r=\max \left\{r_{o}, \max (\tau)\right\}
$$

Note that Eq. (9) allows to compute the current values for $r$ in terms of the current value of $\tau$, which depends explicitly on the current total strains (see Eqs. (1) and (4)).

Finally, the damage index $d=d(r)$ is explicitly defined in terms of the corresponding current value of the damage threshold, so that it is a monotonically increasing function such that $0 \leq d \leq 1$.

In this work, we will use the following functions:

- Linear softening:

$$
d(r)=\left\{\begin{array}{cc}
\left(1+H_{S}\right)\left(1-\frac{r_{o}}{r}\right) & r_{o} \leq r \leq r_{u}=r_{o}\left(1+\frac{1}{H_{S}}\right) \\
1 & r \geq r_{u}
\end{array}\right.
$$

- Exponential softening:

$$
d(r)=1-\frac{r_{o}}{r} \exp \left\{-2 H_{S}\left(\frac{r-r_{o}}{r_{o}}\right)\right\} \quad r_{o} \leq r
$$

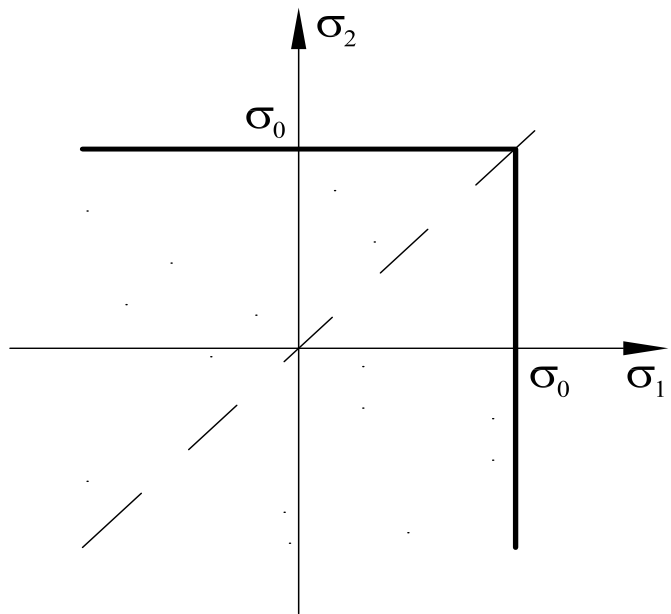

(a)

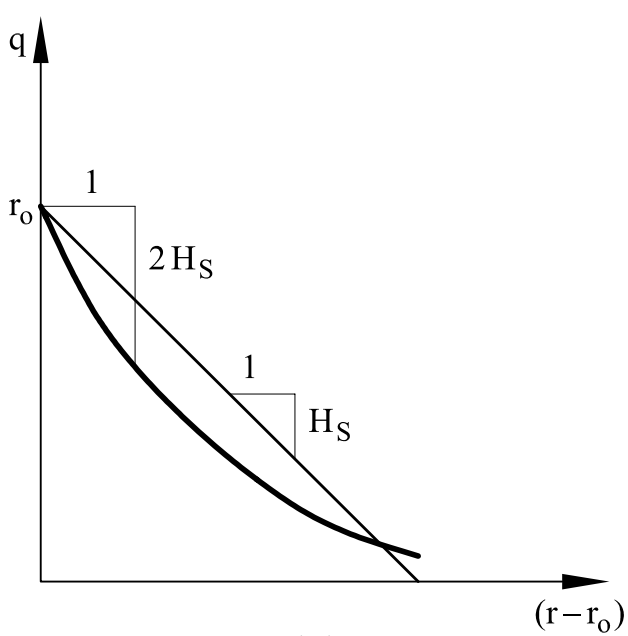

(b)

Figure 2: Rankine damage model: (a) damage surface, (b) softening functions 
where $H_{S} \geq 0$ is a constant.

It is also possible to express the damage laws in the form [41]:

$$
d(r)=1-\frac{q(r)}{r} \quad r_{o} \leq r
$$

where the function $q=q(r)=(1-d(r)) r$ is the stress-like softening function. In this format, the softening laws can be rewritten as

- Linear softening:

$$
q(r)=\left\{\begin{array}{cl}
r_{o}-H_{S}\left(r-r_{o}\right) & r_{o} \leq r \leq r_{u} \\
0 & r \geq r_{u}
\end{array}\right.
$$

- Exponential softening:

$$
q(r)=r_{o} \exp \left\{-2 H_{S}\left(\frac{r-r_{o}}{r_{o}}\right)\right\} \quad r_{o} \leq r
$$

Figure $2 \mathrm{~b}$ shows a schematic representation of both these functions.

\subsection{Mechanical dissipation}

The mechanical free energy term for the damage model is defined in the form:

$$
W=(1-d) W^{e}(\varepsilon)=(1-d)\left[\frac{1}{2} \varepsilon: \mathbf{C}: \boldsymbol{\varepsilon}\right] \geq 0
$$

Thus, the rate of mechanical dissipation can be expressed as

$$
\dot{\mathcal{D}}=W^{e} \dot{d} \quad \geq 0
$$

provided that the damage index increases monotonically, $\dot{d} \geq 0$.

\subsection{Strain-softening and fracture energy release}

Expressions (10) and (11) are able to reproduce the softening branch that occurs in a 1D tensile test after the peak stress is reached, with the tensile stress decreasing to the strain axis, asymptotically in the exponential case. The finite area retained between the stress-strain curve and the strain axis defines the available energy to be dissipated in the control volume during the softening process. If the softening curve and, consequently, this area 
are considered as material properties, FE results necessarily exhibit lack of objectivity, as the strains tend to localize in a band that is only one element across, independently of the element size. Upon mesh refinement, as element size tends to zero, no energy is dissipated in the failure process. Clearly, this is physically unacceptable.

This can be remedied by modifying the softening law in such a way that the energy dissipated over a completely degraded finite element be equal to a given value, which depends on the fracture energy of the material and on the element size [14]. In each element, the computational width of the fracture zone is called the element characteristic length $l_{\mathrm{ch}}([42],[43],[44])$; it is computed depending on the geometric dimensions of the element. The specific dissipated energy $\mathcal{D}$ is then scaled for each element so that the equation

$$
\mathcal{D} l_{\mathrm{ch}}=\mathcal{G}_{f}
$$

holds, where $\mathcal{G}_{f}$ is the mode I fracture energy of the material, regarded to be a material property. This makes the softening modulus $H_{S}$, which defines the softening response, dependent on the element size. It also sets a maximum size for the elements that can be used in the analysis.

The procedure is as follows: consider an ideal uniaxial tensile experiment in which the tensile strain increases monotonically and quasi-statically from an initial unstressed state to another in which full degradation takes place. The specific energy dissipated in the process is:

$$
\begin{aligned}
\mathcal{D} & =\int_{t=0}^{t=\infty} \dot{\mathcal{D}} d t \\
& =\int_{t=0}^{t=\infty} W^{e} \dot{d} d t \\
& =\frac{1}{2 E} \int_{r=r_{o}}^{r=\infty} r^{2} d^{\prime} d r
\end{aligned}
$$

where $E$ is the Young's modulus and we have used Eqs. (16), (??), (1), (4), (9) and the rate of damage has been expressed as $\dot{d}=d^{\prime} \dot{r}$.

We will consider in the following both the cases of linear and exponential softening:

\section{- Linear softening:}

Using Eq. (10), $d^{\prime}=\left(1+H_{S}\right) r_{o} / r^{2}$, for $r_{o} \leq r \leq r_{u}$, with $r_{u}=$ $r_{o}\left(1+1 / H_{S}\right)$, and $d^{\prime}=0$, otherwise. Recalling that $r_{o}=\sigma_{o}$, and 
integrating, we have

$$
\mathcal{D}=\left(1+\frac{1}{H_{S}}\right) \frac{\sigma_{o}^{2}}{2 E}
$$

and equating $\mathcal{D}=\mathcal{G}_{f} / l_{\mathrm{ch}}$, we have

$$
H_{S}=\frac{\bar{H}_{S} l_{\mathrm{ch}}}{1-\bar{H}_{S} l_{\mathrm{ch}}} \quad \geq 0
$$

where $\bar{H}_{S}=\sigma_{o}^{2} /\left(2 E \mathcal{G}_{f}\right)$ depends only on the material properties, as $\mathcal{G}_{f}$ is the mode I fracture energy per unit area, $\sigma_{o}$ is the uniaxial strength and $E$ is the Young's modulus.

- Exponential softening:

Using now Eq. (11), $d^{\prime}=\left(r_{o}+2 H_{S} r\right) \exp \left\{-2 H_{S}\left(\frac{r-r_{o}}{r_{o}}\right)\right\} / r^{2}$, for $r_{o} \leq r \leq \infty$. Recalling that $r_{o}=\sigma_{o}$, and integrating, we obtain an expression which is identical to the result in (21).

Note that in Eq. (22) the specific softening parameter $\bar{H}_{S}$ measures the brittleness of the material, while the elemental softening parameter $H_{S}$ measures the brittleness of the finite element.

It must be remarked that the above computation of the total specific dissipation is only exact for an uniaxial stress state. In a more general case, the total dissipated energy is larger than that in expression (21). This is a consequence of using an isotropic damage model with only one damage index, where the damage due to the straining in one principal stress direction causes degradation in all other directions. Fortunately, it is possible to formulate more sophisticated damage models that lead to a more accurate control of the released elastic energy. For instance, references [45], [46] and [47] present a damage model with two different tension and compression damage indices which greatly overcomes this problem.

In the framework of local models and finite element analysis, the state variables are computed at the integration points in terms of the local strain (and/or stress) history. Therefore, the characteristic length is related to the volume (or area) of each finite element. For linear simplex elements, the characteristic length can be taken as the representative size of the element, $l_{\mathrm{ch}}=h_{e}$. In this work, and assuming that the elements are equilateral, the size of the element will be computed as $h_{e}^{2}=(4 / \sqrt{3}) A_{e}$ for triangular elements, $A_{e}$ being the area of the element, and as $h_{e}^{3}=(12 / \sqrt{2}) V_{e}$ for tetrahedral elements, where $V_{e}$ is the volume of the element. 


\subsection{Tangent operator}

Differentiating Eq. (2) with respect to time, we obtain

$$
\dot{\boldsymbol{\sigma}}=(1-d) \dot{\overline{\boldsymbol{\sigma}}}-\dot{d} \overline{\boldsymbol{\sigma}}
$$

The effective stresses $\overline{\boldsymbol{\sigma}}$ are computed in terms of the total strain tensor $\boldsymbol{\varepsilon}$ as

$$
\bar{\sigma}=\mathrm{C}: \varepsilon
$$

where $\mathbf{C}$ is the usual (fourth order) linear-elastic isotropic constitutive tensor. Differentiating this with respect to time, we have

$$
\dot{\bar{\sigma}}=\mathrm{C}: \dot{\varepsilon}
$$

Despite the simplicity of the stress split postulated in Eq. (3), which expresses $\overline{\boldsymbol{\sigma}}^{+}$in terms of the (positive) eigenvalues and eigenvectors of $\overline{\boldsymbol{\sigma}}$, quite more involved operations are required to express $\dot{\overline{\boldsymbol{\sigma}}}^{+}$in terms of $\dot{\overline{\boldsymbol{\sigma}}}$. It can be shown that the appropriate expressions are [46]

$$
\dot{\overline{\boldsymbol{\sigma}}}^{+}=\mathbf{P}: \dot{\overline{\boldsymbol{\sigma}}}=\mathbf{P}: \mathbf{C}: \dot{\boldsymbol{\varepsilon}}
$$

where the projection operator $\mathbf{P}$ is

$$
\mathbf{P}=\sum_{i=1}^{3} H\left(\bar{\sigma}_{i}\right) \mathbf{P}^{i i} \otimes \mathbf{P}^{i i}+2 \sum_{\substack{i, j=1 \\ j>i}}^{3} \frac{\left\langle\bar{\sigma}_{i}\right\rangle-\left\langle\bar{\sigma}_{j}\right\rangle}{\bar{\sigma}_{i}-\bar{\sigma}_{j}} \mathbf{P}^{i j} \otimes \mathbf{P}^{i j}
$$

where $H(\cdot)$ is the Heaviside function, $\langle\cdot\rangle$ are the Macaulay brackets and

$$
\mathbf{P}^{i j}=\mathbf{P}^{j i}=\frac{1}{2}\left(\mathbf{p}_{i} \otimes \mathbf{p}_{j}+\mathbf{p}_{j} \otimes \mathbf{p}_{i}\right)=\operatorname{symm}\left(\mathbf{p}_{i} \otimes \mathbf{p}_{j}\right)
$$

On the other hand, recalling from the previous section that the rate of the damage index can be expressed as

$$
\dot{d}=d^{\prime} \dot{r}
$$

where the first derivative term can be obtained from Eqs. (10) or (11). On loading, consistency requires that $\dot{r}=\dot{\tau}$, and therefore, differentiating Eq. (5), we can write

$$
\begin{aligned}
\dot{r} & =\dot{\tau} \\
& =\frac{1}{\tau}\left[\overline{\boldsymbol{\sigma}}^{+}: \boldsymbol{\Lambda}: \dot{\overline{\boldsymbol{\sigma}}}^{+}\right] \\
& =\frac{1}{\tau}\left[\overline{\boldsymbol{\sigma}}^{+}: \mathbf{\Lambda}: \mathbf{P}: \mathbf{C}: \dot{\boldsymbol{\varepsilon}}\right]
\end{aligned}
$$


On unloading, it is $\dot{r}=0$. Substituting this result in Eq. (29), and the result in Eq. (23), jointly with Eqs. (24) and (26), yields the desired expression

$$
\dot{\boldsymbol{\sigma}}=\mathbf{C}^{\tan }: \dot{\varepsilon}
$$

with

$$
\mathbf{C}^{\tan }=\left[(1-d) \mathbf{I}-h\left(\overline{\boldsymbol{\sigma}}^{+} \otimes \overline{\boldsymbol{\sigma}}^{+}\right): \mathbf{\Lambda}: \mathbf{P}\right]: \mathbf{C}
$$

where the coefficient $h$ is

$$
h=\left\{\begin{array}{cl}
\frac{d^{\prime}}{\tau} & \text { for loading } \\
0 & \text { for unloading }
\end{array}\right.
$$

Note that the tangent tensor in Eq. (32) is, in general, nonsymmetric. This is often inconvenient for practical finite element applications, as it results in a nonsymmetric tangent stiffness matrix. In those cases, and at the cost of the loss of rate of convergence, the tangent matrix can be replaced with the secant matrix, computed with the secant constitutive tensor

$$
\mathbf{C}^{\mathrm{sec}}=(1-d) \mathbf{C}
$$

which is much simpler to compute and always symmetric.

\subsection{Final remarks}

Let us close this section about constitutive modelling with three remarks about isotropic continuum damage and strain softening.

In the FM community a technique known as "element extinction" is sometimes used. This consists in simply deleting from the FE mesh those elements lying along the crack path. The results obtained are satisfactory if the finite element mesh used is fine enough. In a CM framework, such "extinction" must be done with care, that is, taking into account the elastic energy released when performing it. This is, precisely, what an isotropic damage accomplishes: when the damage index reaches its final value, $d=1$, the totally degraded element is effectively removed from the mesh; but this process takes place gradually, and while it is occurring the elastic energy is released at a rate determined by the brittleness of the particular element. 
The second remark is about using isotropic models to reproduce a phenomenon like tensile cracking, which is actually directional. This choice implies that the macroscopic anisotropy of the structural behaviour has to be captured by means of the finite element approximation to within the resolution of the adopted mesh ([37], [45], [48]). The use of orthotropic models, like the now old-fashioned, although still very popular, fixed and rotating smeared crack models of the 1980's presents serious stress locking problems, reported but unsolved. The origin of this locking difficulties undoubtedly lies in the inflexibility of the spatial discretization used.

The third remark is about the concept of strain softening itself. It is often argued, particularly from the fracture mechanics community, that a material with negative tangential moduli is not a sound concept, as such material would be unstable and would not propagate waves. This may be true, but the fact that the constitutive model, formulated in terms of nominal stresses and strains, contemplates strain softening does not mean that strain softening needs to have physical meaning. Damage models evaluate the stresses as an area weighted average of the stresses acting on virgin material and on voids or defects. To do this, they take into account the surface density of defects in the material, which is, by concept [49], the damage index. In this sense, the behaviour of the softening damaged material upon straining has perfect meaning as an average of the non-softening virgin material and the growing density of defects that are developing inside it.

\section{Boundary value problem}

\subsection{Strong and weak forms}

The strong form of the continuum mechanical problem can be stated as: find the displacement field $\mathbf{u}$, for given prescribed body forces $\mathbf{f}$, such that:

$$
\nabla \cdot \boldsymbol{\sigma}+\mathbf{f}=\mathbf{0} \quad \text { in } \Omega
$$

where $\Omega$ is the open and bounded domain of $\mathbb{R}^{n_{\text {dim }}}$ occupied by the solid in a space of $n_{\text {dim }}$ dimensions. Eq. (35) is subjected to appropriate Diritchlet and Neumann boundary conditions. In the following, we will assume these in the form of prescribed displacements $\mathbf{u}=\overline{\mathbf{u}}$ on $\partial \Omega_{u}$, and prescribed tractions $\overline{\mathbf{t}}$ on $\partial \Omega_{t}$, respectively. 
Following the standard procedure, the corresponding discrete weak problem is

$$
\left(\nabla^{s} \mathbf{v}_{h}, \boldsymbol{\sigma}_{h}\right)-\left(\mathbf{v}_{h}, \mathbf{f}\right)-\left(\mathbf{v}_{h}, \overline{\mathbf{t}}\right)_{\partial \Omega_{t}}=0 \quad \forall \mathbf{v}_{h}
$$

where $\mathbf{v}_{h} \in \mathcal{V}_{h}$ are the variations of the displacement field $\mathbf{u}_{h}, \mathcal{V}_{h}$ is a subspace of $H^{1}(\Omega)$, that is, the space of functions square integrable in $\Omega$ with square integrable derivatives; $(\cdot, \cdot)$ denotes the inner product in $L^{2}(\Omega)$.

This discrete problem is nonlinear because of the dependence of the stresses $\boldsymbol{\sigma}_{h}$ on the displacements $\mathbf{u}_{h}$. In practice, this nonlinearity is dealt with assuming that the acting body forces and boundary tractions, $\mathbf{f}$ and $\overline{\mathbf{t}}$, are applied incrementally, being dependent on (pseudo)time or other loading parameter. Then, the problem is solved step-by-step in time (or load), and iterating within each step until equilibrium (Eq. (36)) is satisfied.

\subsection{Stability and well-posedness}

Over the last years, many researchers have supported the idea that the underlying reason why the standard, local, rate-independent constitutive models are inadequate to model localized straining correctly is the local change of character of the governing equations (see, for instance, [17], [18], [19], [20], [21], [22], [23], [24]). Let us consider this question by considering both the continuum problem and the discrete problem.

Let us start the discussion on the continuum problem considering the case of standard elasticity, with a non-uniform distribution of elastic moduli. The irreducible governing Eq. (35) can be rewritten in terms of the deviatoric and volumetric parts of the deformation as

$$
\nabla \cdot\left(G \nabla^{s} \mathbf{u}\right)+\nabla(K \nabla \cdot \mathbf{u})+\mathbf{f}=\mathbf{0} \quad \text { in } \Omega
$$

where $G$ and $K$ are the shear and bulk moduli, respectively.

A standard stability (or energy) estimate for problem (37) is obtained by multiplying the first two terms of the left hand side by $\mathbf{u}$ and integrating by parts over the domain $\Omega$, to yield

$$
\left(\nabla^{s} \mathbf{u}, G \nabla^{s} \mathbf{u}\right)+(\nabla \cdot \mathbf{u}, K \nabla \cdot \mathbf{u})=\|\mathbf{u}\|_{E}^{2}>0
$$

where $\|\cdot\|_{E}^{2}$ is the energy norm (equal to the elastic free energy). For strictly positive elastic moduli, $G, K>0$, the stability of the elastic governing equation is evident. 
For an isotropic damage constitutive model, the stability estimate reads

$$
\left(\nabla^{s} \mathbf{u}, G_{\mathrm{sec}} \nabla^{s} \mathbf{u}\right)+\left(\nabla \cdot \mathbf{u}, K_{\mathrm{sec}} \nabla \cdot \mathbf{u}\right)>0
$$

where stability can be guaranteed as long as the secant moduli, $G_{\mathrm{sec}}=(1-$ d) $G$ and $K_{\mathrm{sec}}=(1-d) K$, remain strictly positive, that is, for damage index $d<1$. Therefore, in the problem of nonlinear solid continuum mechanics with softening, the governing equation in terms of the total displacement $\mathbf{u}$ (not the rate equation, written in terms of the incremental displacements) remains stable as long as the secant moduli remain strictly positive.

Upon continuing straining, the damage index approaches 1 and the secant moduli may eventually vanish. However, inequality (39) still holds if the secant moduli vanish completely only in a subdomain $S \subset \Omega$ of zero measure. This would be the case of a line crack in $2 \mathrm{D}$ or a surface crack in 3D. Anyhow, this indicates the possible origin of difficulties in the extension and propagation of the areas where stiffness is completely lost.

For the discrete problem to be stable, it must hold

$$
\left(\nabla^{s} \mathbf{u}_{h}, G_{\mathrm{sec}} \nabla^{s} \mathbf{u}_{h}\right)+\left(\nabla \cdot \mathbf{u}_{h}, K_{\mathrm{sec}} \nabla \cdot \mathbf{u}_{h}\right)>0
$$

where now $\mathbf{u}_{h}$ represents the discrete displacement field. Stability can be maintained if $\mathbf{u}_{h}$ is discontinuous, ensuring that the secant moduli vanish completely only in a subdomain of zero measure in $\Omega$. However, condition (40) also holds if the secant moduli vanish only in a properly restricted subdomain in $\Omega$, such as in a band of elements (one element across) overlapping the crack. This opens the possibility of solving crack propagation problems using standard elements with continuous displacement fields $\mathbf{u}_{h}$, if the extension of the totally damaged areas is restricted to a band.

Let us now approach the question of well-posedness in a more empirical way. For the sake of discussion, let us imagine that we proceed to solve Eq. (36) by means of an incremental procedure, advancing in (pseudo)time, and using sufficiently small time steps of size $\Delta t$, in order to rule out of the discussion the associated time discretization error. For each time step we proceed in two stages: in the first stage, we solve for the displacement field at time $t$ in the domain with the distribution of damage "frozen" at the previous time step, $t-\Delta t$; in the second stage, we update the damage distribution according to the strain field computed in the previous stage. This second stage would involve two different operations: (i) the updating of the damage 
index of those elements already damaged in the previous time steps and (ii) deciding which elements are newly damaged during the current time step.

This purely incremental procedure may not seem natural in the context of non linear continuum mechanics, where equilibrium iterations are performed for each time step and operations (i) and (ii) are done concurrently. However, this is the procedure used in fracture mechanics to propagate a crack: first stage, solve the problem for a given crack path and, second stage, update the crack path, by advancing the crack tip a small distance, according to the selected (empirical) criterion for crack propagation.

Observe now the implications of proceeding in this way. The first stage (at frozen damage) consists of solving a linear elastic BVP, with a given distribution of (positive) elastic moduli. Note that this stage can be solved evaluating only the secant moduli, although this would correspond to a first order linearization of the original nonlinear problem. Thus, the problem is obviously linear, well-posed, elliptic, stable and the solution is unique. In the second stage, updating the damage level for those elements already damaged in previous time steps is straight-forward, as damage is an explicit function of the strain field. Therefore, all the difficulties reside in deciding which elements are newly damaged during the current time step. In principle, this should not be a problem, as the damage criterion is also unambiguously written in terms of the total strain. But, it turns out that the computed damage distribution is "incorrect", as it depends spuriously on the alignment of the finite element mesh. The reason for this must be that the computed strain distribution in the vicinity of the advancing front of damaged elements (what we could consider the "tip of the crack") is mesh-biased. In fact, in the continuum problem the tip of the crack is a singular point and, therefore, the $L_{\infty}$-norm of the error on the displacement gradients (strains) in the computed discrete solution is unbounded.

We may conclude that the main difficulty in solving the problem of tensile crack propagation using standard elements, with continuous displacement fields and local constitutive models, with properly size-adjusted strainsoftening, is the approximation error due to the spatial discretization.

If the question of crack propagation is not an issue in the problem at hand, there is no difficulty at all. For instance, consider the 1D problem of a straight bar under tensile straining, with a small defect located at a given position inside the bar. Obviously, the only reasonable solution is a crack initiating and progressively opening at the location of the defect. If the problem is solved with small enough time increments so to ensure that only 
the finite element containing the defect opens at the proper time step, and the element size is adequately taken into account to adjust the local softening, the global response of the bar, in terms of load vs. end displacement is unique and mesh-size objective.

\section{The problem of crack propagation}

\subsection{The convenience of crack tracking}

In the discrete crack approach, the two basic ingredients of the formulation are: (a) the criterion for crack propagation, which is always established in terms of the stored elastic energy, and (b) the criterion for selecting the $d i$ rection of crack propagation, which is established empirically. Here, several possibilities have been formulated [50]: the principal tensile stress direction, the maximum circumferential stress direction and the direction that maximizes the strain energy release rate, etc.

In any case, the discrete crack approach requires the careful tracking of the propagation of the crack through the FE mesh. Tracking algorithms are always an essential part of FM based codes, as they are in the application of the X-FEM.

On the other hand, in the smeared crack approach it has always been implicitly assumed that the criterion for the onset of cracking, which is always established in terms of stresses/strains, also must automatically define the direction of propagation. This may be a natural assumption in the continuum problem, with proper evaluation of stress and strain values and directions. However, in the discrete problem the stress and strain fields evaluated in the vicinity of the crack tip differ greatly from being exact. As a consequence, the automatic application of the cracking criterion for the evaluation of the direction of crack growth leads to an unacceptable dependence on the mesh bias in this region. This local error must be overcome if reasonable solutions are to be obtained with the smeared crack approach.

In the last decade, the so-called strong discontinuity approach has been developed as a continuum mechanics alternative to the fracture mechanics formulation. Remarkably, and although it has not been always explicitly stated, successful applications of this approach also use tracking algorithms to determine the direction of crack propagation. In fact, Mosler and Meschke [32] have reported that if tracking is not used, the strong discontinuity for- 
mulation leads to the same spurious mesh bias dependence as the standard weak discontinuity approach.

All this evidence point to the potential advantages of using a crack tracking algorithm in the discrete format of the crack propagation problem, also if continuous displacement fields are used in the interpolation basis.

\subsection{Tracking algorithms}

Two requirements can be stated for a tracking algorithm to be successfully used in crack propagation problems: (1) it must be consistently linked to the cracking criterion, as this is the established cracking mechanism at continuum level, and (2) it should not be completely dependent on the local values of the discrete stress/strain fields, as these may be substantially off-track.

With regard to the first requirement, for a Rankine criterion based on the value of the maximum tensile principal stress, it is consistent to assume that the crack propagates in the plane orthogonal to the corresponding first stress eigenvector. Regarding the second requirement, several possibilities are feasible, and at least two have been proposed and successfully applied.

The first one is to apply a stress (or strain) recovery procedure to improve the stress (or strain) fields computed in the vicinity of the crack tip. This is readily done by applying smoothing techniques [51], from simple averaging among adjacent elements to a more refined patch-based interpolation.

The second, more fundamental, approach is to consider the evaluation of the propagation direction as a separate problem, obviously coupled to that of solving the balance of momentum equation (36). This procedure was proposed in reference [30] in the strong discontinuity framework, and it has been already used in $2 \mathrm{D}$ and $3 \mathrm{D}$ applications [31]. In this work we will use this second strategy in the context of standard finite elements. The implementation of this technique is described in the following section.

\subsection{Evaluation of the propagation direction}

Let us assume that the crack propagates following a surface (a line in 2D) which is orthogonal to the direction of the maximum positive principal stress. Then, to be able to predict the direction of propagation of the crack it is necessary to evaluate the principal stress trajectories in the vicinity of the crack tip. This can be accomplished in the following way. 
For a given time, let $\mathbf{n}$ be a field of unit vectors in the direction of the maximum positive principal stress at each point of the domain $\Omega$ and $\mathbf{s}$ and $\mathbf{t}$ be any two orthogonal unit vectors orthogonal to it. Let $\theta$ be a scalar field such that its gradient is parallel to the given vector field $\mathbf{n}$, so that $\mathbf{n}=\nabla \theta /\|\nabla \theta\|$. It is clear that the iso-level surfaces (lines in 2D) defined by $\theta=$ const are orthogonal to $\mathbf{n}$. Therefore, the crack propagates along one particular iso-level surface $S$ defined by $\theta=\bar{\theta}_{o}$. Thus, the problem of evaluating the direction of crack propagation is equivalent to finding the scalar field $\theta$ and determining the iso-level locus $\theta=\bar{\theta}_{o}$.

This can be formulated as the following linear BVP: find the scalar field $\theta$, such that:

$$
\nabla \cdot(\mathbf{K} \cdot \nabla \theta)=0 \quad \text { in } \Omega
$$

where $\Omega$ is the open and bounded domain of $\mathbb{R}^{n_{\text {dim }}}$ occupied by the solid in a space of $n_{\text {dim }}$ dimensions.

Eq. (41) is subjected to appropriate boundary conditions. Let $\mathbf{x}_{o}$ be the point of the boundary where the crack is initiated and $\bar{S} \subset S$ be the part of the surface $S$ where the cracking criterion has already been violated (consolidated part of the crack). Dirichlet boundary conditions are specified in (a) a part of the boundary $\partial \Omega_{\theta} \subset \partial \Omega$ including the seminal point $\mathbf{x}_{o} \in \partial \Omega_{\theta}$, and so that $\theta\left(\mathbf{x}_{o}\right)=\bar{\theta}_{o}$ and (b) along $\bar{S}$, so that $\theta(\mathbf{x})=\bar{\theta}_{o}$ for points $\mathbf{x} \in \bar{S}$; natural boundary conditions are imposed elsewhere at $\partial \Omega$ (see Fig. 3).

The second-order tensor $\mathbf{K}$ couples the scalar problem (41) to the evolution of the mechanical problem, Eq. (35). It takes the form

$$
\mathbf{K}=\mathbf{t} \otimes \mathbf{t}+\mathbf{s} \otimes \mathbf{s}+\varepsilon \mathbf{n} \otimes \mathbf{n}
$$

where $\varepsilon$ is a small perturbation value, $\varepsilon=10^{-4} \div 10^{-10}$. This enforces that $\mathbf{n}=\nabla \theta /\|\nabla \theta\|$.

The corresponding discrete weak problem is

$$
\left(\mathbf{K} \cdot \nabla \theta_{h}, \nabla \eta_{h}\right)=0 \quad \forall \eta_{h}
$$

where $\eta_{h} \in \mathcal{Q}_{h} \subset H^{1}(\Omega)$ are the variations of the scalar field $\theta_{h}$.

Problem (43) is linear, elliptic and it only involves one unknown per node. Besides, being a conduction-like problem, it is sufficiently well-behaved and it does not present any singular point in the vicinity of the advancing crack. It can be solved using the same FE mesh as problem (36) and the coupling with it can be enforced once per time increment or, more rigorously, at each 


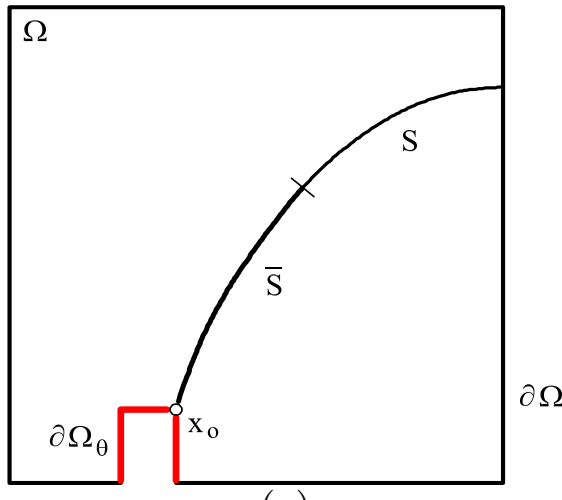

(a)

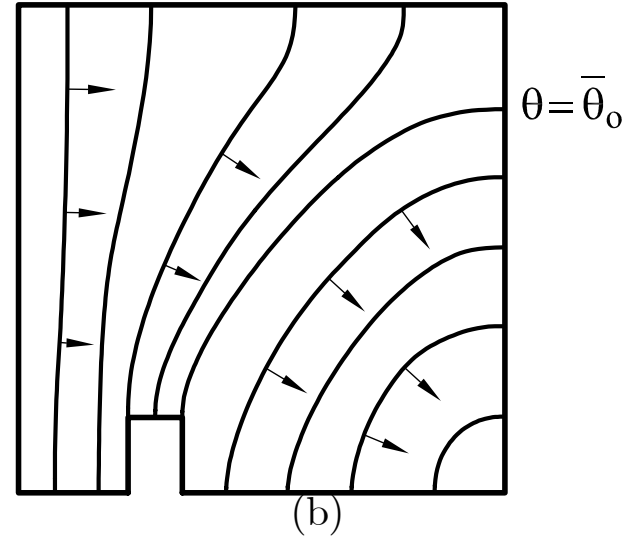

(b)

Figure 3: Tracking algorithm: (a) definition, (b) iso-level curves

iteration. Once it is solved, and the elements $e$ crossed by the iso-level locus $S$, such that $\theta=\bar{\theta}_{o}$, are identified, these are subsequently known to the mechanical solver when performing the check on the crack criterion; only those elements crossed by $S$ are allowed to crack, and those actually cracked are added to the consolidated part of the track $\bar{S} \subset S$. From then on, the corresponding boundary condition is imposed at the nodes pertaining to those elements.

The described algorithm can be easily extended to track the propagation of multiple cracks, simply by defining the $i-t h$ crack as the locus $S^{i}$ where $\theta=\bar{\theta}_{o}^{i}$ and specifying the corresponding boundary conditions at $\bar{S}^{i} \subset S^{i}$.

Implementation of equation (43) is straightforward in a standard FE framework, and it becomes trivial in those FE codes intended for coupled multifield formulations, such as thermo-mechanical or seepage-mechanical problems.

\section{$5 \quad$ Numerical examples}

The formulation presented in the preceding sections is illustrated below by solving two different benchmark problems. Performance of the standard continuous displacement finite elements is tested considering $2 D$ plane-strain 3noded linear triangular meshes. The poor behaviour of the linear simplex in some particular situations, such as pure bending or quasi-incompressibility is well-known, but this does not affect the following tests. 
The examples are solved using the continuum isotropic damage model presented in Section 2 with exponential softening, adjusted according to the element size. The following material properties are assumed for both examples: Young's modulus $E=30 \mathrm{MPa}$, Poisson's ratio $\nu=0.2$, tensile strength $\sigma_{o}=2 \mathrm{KPa}$ and mode I fracture energy $G_{f}=100 \mathrm{~J} / \mathrm{m}^{2}$.

The discrete problem is solved incrementally, in a (pseudo)time step-bystep manner. In all cases 200 equal time steps are performed to complete the analyses. Within each step, a modified Newton-Raphson method (using the secant stiffness matrix), together with a line search procedure, is used to solve the corresponding non-linear system of equations. Convergence of a time step is attained when the ratio between the norm of the iterative and the incremental norm of the computed displacements is lower than $1 \%$.

Calculations are performed with an enhanced version of the finite element program COMET [52], developed by the authors at the International Center for Numerical Methods in Engineering (CIMNE). Pre and post-processing is done with GiD, also developed at CIMNE [53].

\subsection{Perforated strip under tension}

The first example is a plane-strain perforated strip subjected to axial vertical straining imposed at both ends. Because of the symmetry of the domain and boundary conditions, only one half of the domain (the right half) needs to be considered. Dimensions of the strip are $20 \times 40 \mathrm{~cm} \times \mathrm{cm}$ (width $\times$ height) and the radius of the perforation is $r=1 \mathrm{~cm}$. This example is selected because the initial geometry does not present any singular point; tensile stresses are larger in the vicinity of the perforation and damage starts there. Also, it represents an example of pure mode I fracture.

The computational domain is discretized in two different unstructured meshes with average mesh sizes of $h_{e}=5 \mathrm{~mm}$ (2,023 nodes) and $h_{e}=2.5$ $\mathrm{mm}(7,648$ nodes). The central part of the two meshes is shown in Fig. 4. It can be seen that the pre-processor used tends to introduce patches of equilateral triangles with predominant directions at $-30^{\circ},+30^{\circ}$ and $+90^{\circ}$ with the horizontal axis.

Two separate analyses are performed using both meshes. The computed deformed shapes of the strip in the vicinity of the perforation are shown in Figures 4a.1 and 4b.1, respectively ( (half)-imposed vertical displacement $\delta=0.1 \mathrm{~mm}$, with a displacement amplification factor of 100; the other half-imposed displacement is applied at the opposite end of the strip). The 


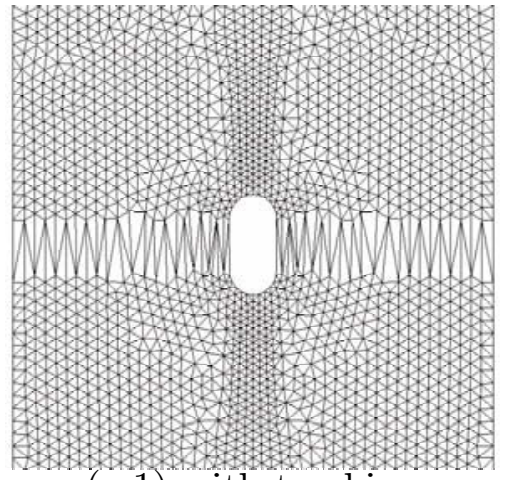

(a.1) with tracking

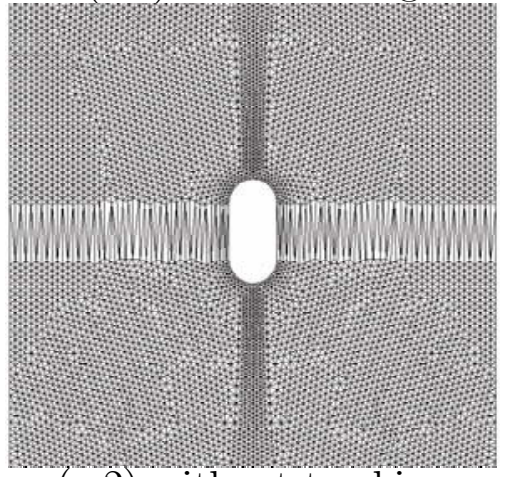

(a.2) without tracking

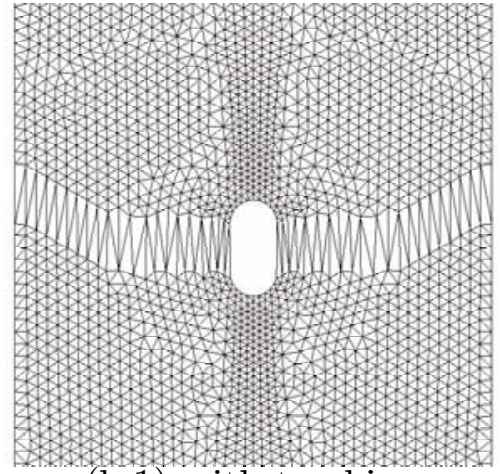

(b.1) with tracking

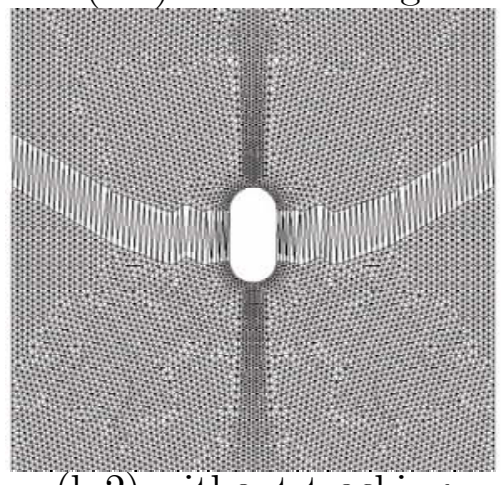

(b.2) without tracking

Figure 4: Deformed geometries (x 100) on the central part of the two meshes with and without tracking for perforated strip

different element sizes in both meshes can be appreciated in these figures. As shown, the computed cracks in both analyses follow exactly the horizontal axis of symmetry of the perforation, even if the elements in neither of the two meshes are aligned along this line. If no tracking strategy is used, see Figures 4a.2 and 4b.2, the crack initiates horizontally in both meshes, but it soon departs from this course to spuriously follow a line of elements along the mesh bias $\left(+30^{\circ}\right.$ in this case).

Figure 5 shows (half)-load vs (half)-imposed vertical displacement curves obtained in the two analyses. Because in this example the strain field is almost uniform prior to the inception of the cracks, the response curve is almost linear until the cracks form in a rather explosive manner, with a nearly exponential softening branch after the limit load is attained.

Note that the overall global response is satisfactorily similar upon mesh 


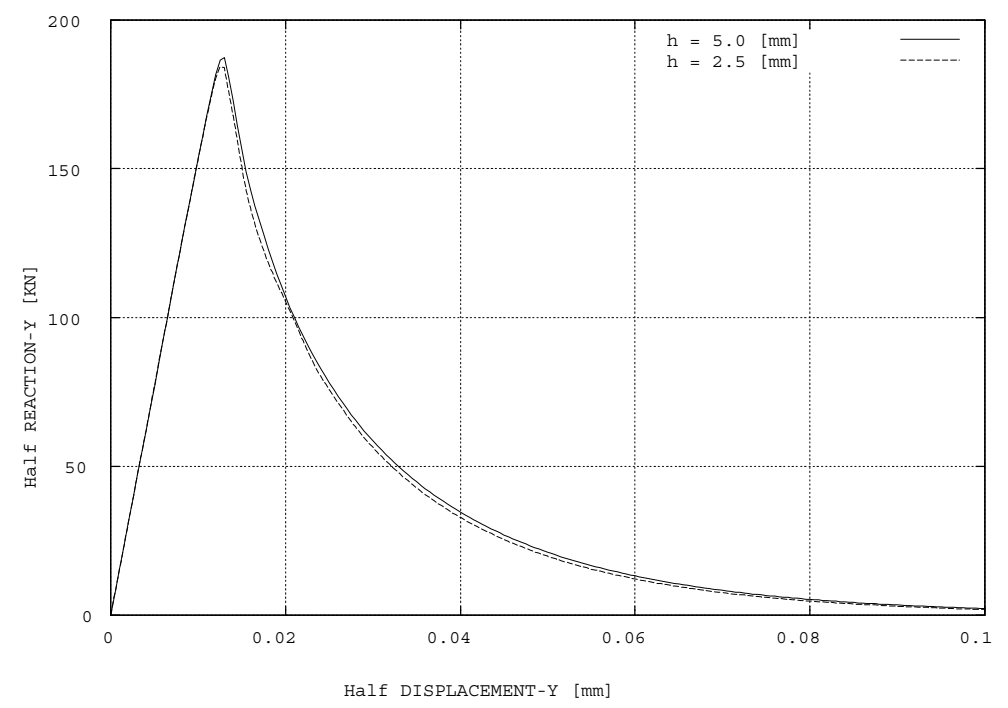

Figure 5: Load versus displacement for perforated strip. Comparison between different mesh sizes

refinement, with the total area under the load-displacement curve converging to the correct amount of energy dissipated to create the cracks. This should be equal, for half of the domain, to $\mathcal{D}^{\text {tot }}=\mathcal{G}_{f} \times l_{\text {cr }} \times t=100 \times 0.09 \times 1=9$ $\mathrm{J}$, where $l_{\text {cr }}$ is the length of the crack $(9 \mathrm{~cm})$ and $t$ is the thickness $(1 \mathrm{~m})$. The area under the curves is almost exactly, half of this value. No spurious brittleness is observed when the size of the elements is reduced.

Figure 6 shows the results obtained using the proposed formulation on the fine mesh. The three columns represent, respectively, the evolution, at four different time steps of the analysis, of the: (a) contours of vertical displacements, (b)contours of maximum principal strain and (c) max. principal strain vectors. The progressive concentration of the displacement gradients (strains) in the elements lying along the horizontal axis of symmetry is evident in the three columns. The bottom figures show how, when the failure mechanism is fully developed, all the deformation concentrates in the formed horizontal crack, while the elements outside this localization band are mostly undeformed. Therefore, the resolution of the cracks is optimal for the mesh used. In the third column, it can be observed that the correct failure mechanism has been predicted although the directions of the computed maximum 

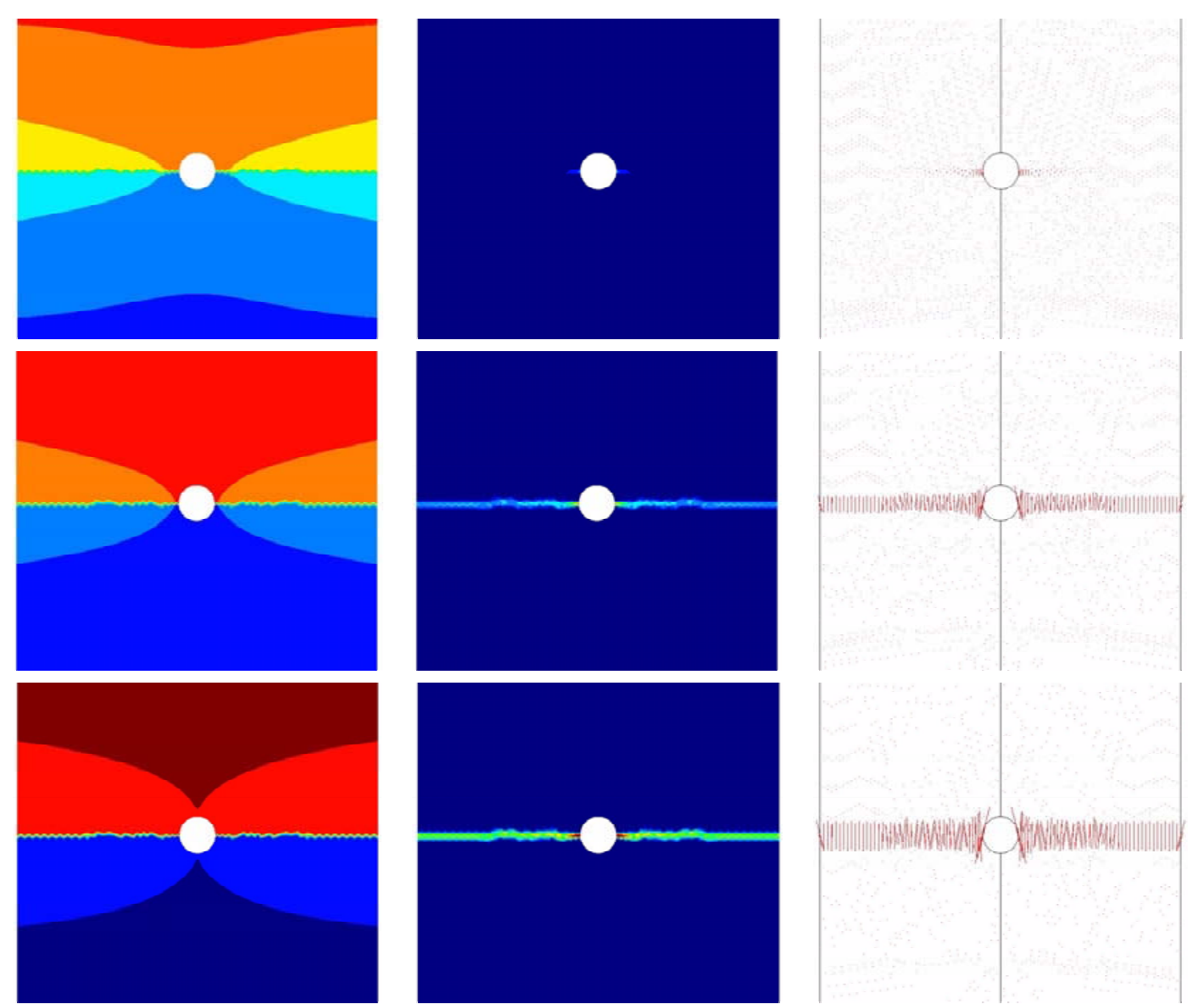

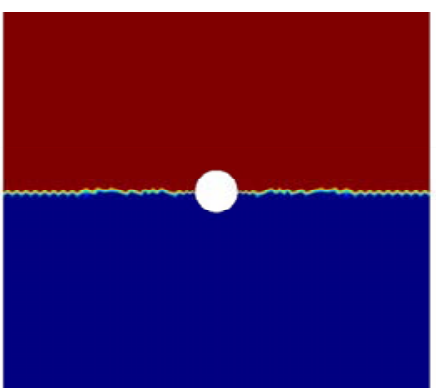

(a)

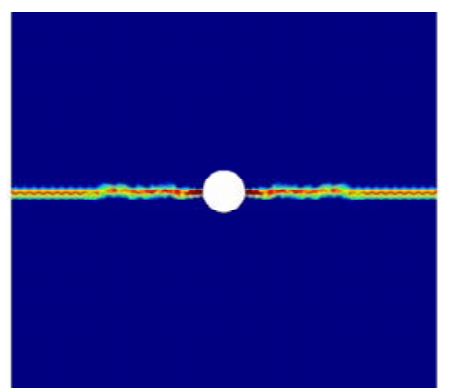

(b)

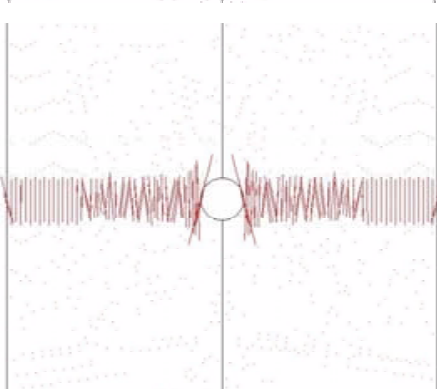

(c)

Figure 6: Results for perforated strip using the proposed formulation. Evolution of: (a) vertical displacement, (b) maximum principal strain, (c) vectors of max. principal strain 
principal strain vectors (as the related vectors of maximum principal effective stress) are clearly dependent on the mesh bias, as they are not vertical everywhere. For the coarser mesh, similar results are obtained, although the strain localization is smeared across a row of elements twice as large.

Finally, Figures $7 \mathrm{a}$ and $7 \mathrm{~b}$ show the evolution, at three different time steps of the analysis, of: (a) the vertical displacements, (b) the maximum principal strain, along a vertical line orthogonal to the formed crack. In the first one, the initially uniform gradient of displacements progressively localizes into a very sharp jump across one single element. In the second one, the strain profile progressively localizes with very sharp resolution.

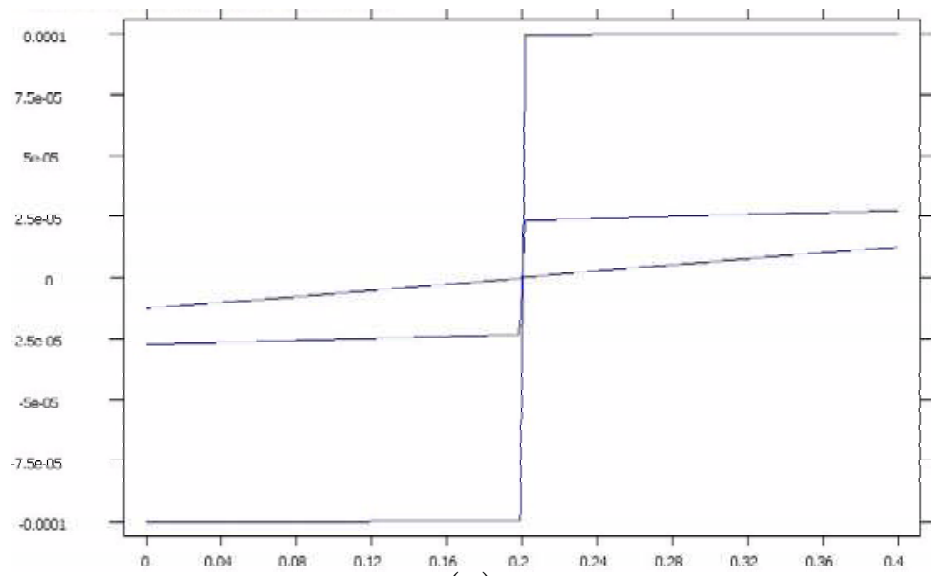

(a)

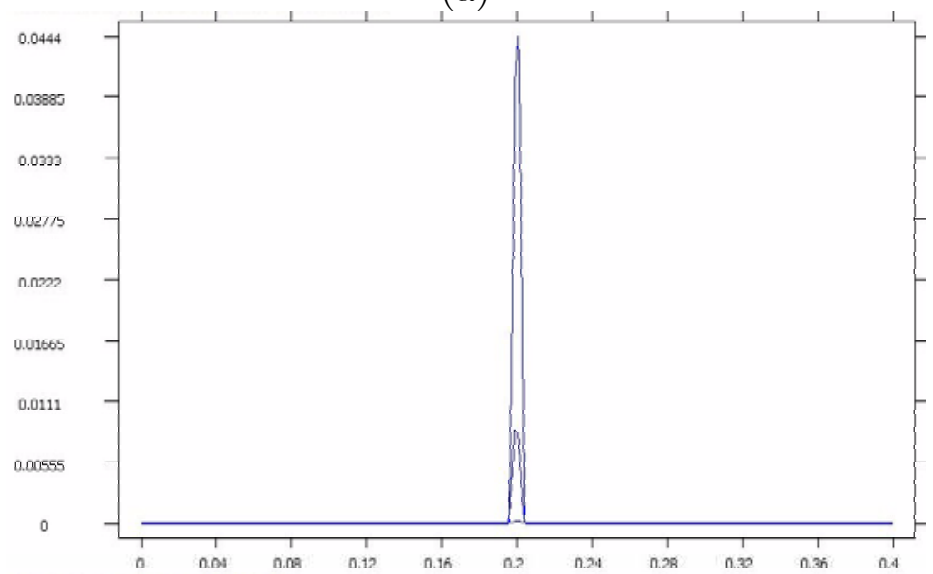

(b)

Figure 7: Evolution of the profiles along a vertical line of: (a) vertical displacement and (b) maximum principal strains for perforated strip 


\subsection{Four point bending beam}

The second example is a plane-strain doubly notched beam subjected to four point bending. Figure 8a depicts the geometry of the problem; dimensions of the beam are $134.0 \times 30.6 \mathrm{~cm} \times \mathrm{cm}$ (width $\times$ height) and the length and width of the notches are $8.2 \mathrm{~cm}$ and $0.5 \mathrm{~cm}$, respectively. The load is applied at the central (rigid) supports (at $8.0 \mathrm{~cm}$ from the center of the beam) by imposing vertical displacements of opposite sign at the top and bottom supports. The two supports near the extremes of the beam (at $20.3 \mathrm{~cm}$ ) are fixed. This example is selected because it presents two singular points at the tips of the notches; tensile stresses are very large in the vicinity of these regions and damage starts there. Also, it represents an excellent example of mixed mode fracture.

The computational domain is discretized in three different unstructured meshes with average mesh sizes of $h_{e}=20 \mathrm{~mm}$ (1,189 nodes), $h_{e}=10 \mathrm{~mm}$ (2,217 nodes) and $h_{e}=5 \mathrm{~mm}$ (5,909 nodes). The central part of the three meshes is shown in Fig. 9.

Three separate analyses are performed using these meshes. The computed deformed shapes of the central part of the beam are shown in Figures 9a.1, 9b.1 and 9c.1, respectively (imposed vertical displacement $\delta=0.1 \mathrm{~mm}$, with a displacement amplification factor of 100). The different element sizes in the

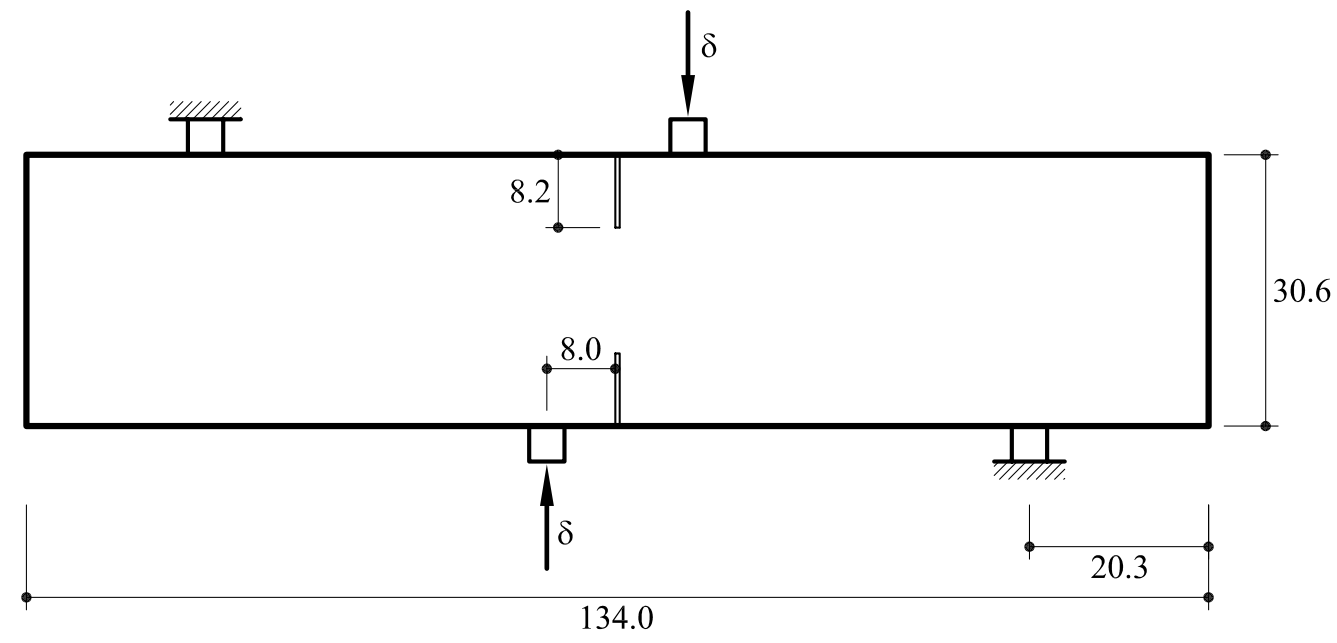

Figure 8: Geometry and load for four point bending beam 


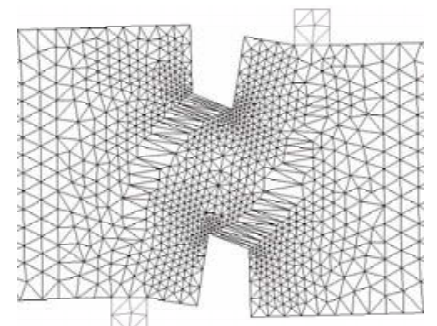

(a.1) with tracking

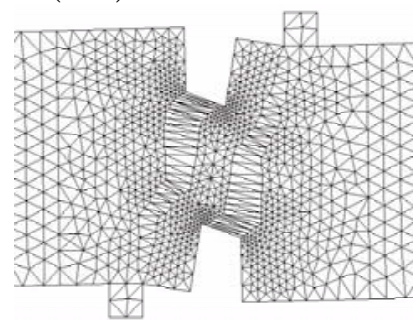

(a.2) without tracking

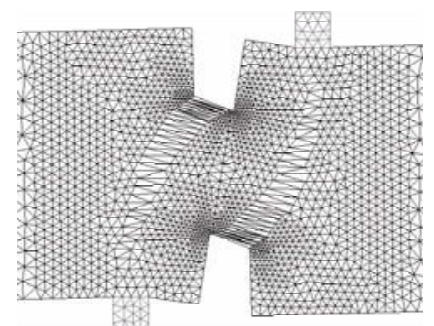

(b.1) with tracking

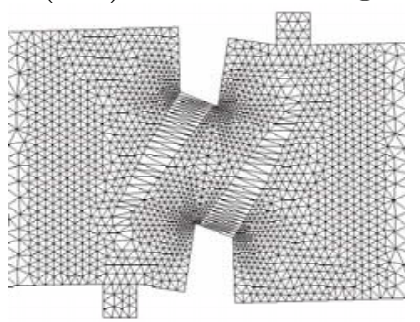

(b.2) without tracking

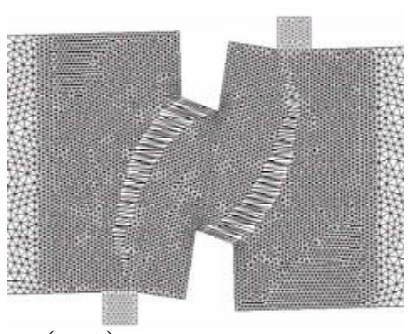

(c.1) with tracking

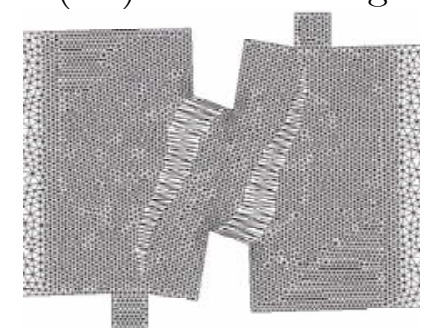

(c.2) without tracking

Figure 9: Deformed geometries (x 100) on the three meshes with and without tracking for four point bending beam

meshes can be appreciated in these figures. As shown, the computed cracks in all the analyses follow very closely the same path, starting at the tip of the notches and turning upwards to the point of application of the loads. No spurious mesh bias is observed in any of the meshes.

If no tracking strategy is used, see Figures 9a.2, 9b.2 and 9c.2, the crack initiates correctly in all cases, but they turn upwards almost immediately to run along with the respective mesh alignment and too close to the notches.

Figure 10 shows load vs imposed vertical displacement curves obtained in the three analyses. In this example the loading branch curves slowly as the cracks progress, turning into the softening branch once the failure mechanism is fully developed. The load does not vanish completely because only damage due to tensile effective stresses is considered, and the state of stresses near the loading supports is mostly compressive.

The overall global response is very similar upon mesh refinement, although the effect of the different spatial discretizations can be observed even in the global elastic stiffness of the beam. This shows that solving problems involving singular stress points requires a high level of resolution.

The total area under the load-displacement curves represents the correct amount of energy necessary to create the cracks. This should be equal, for 


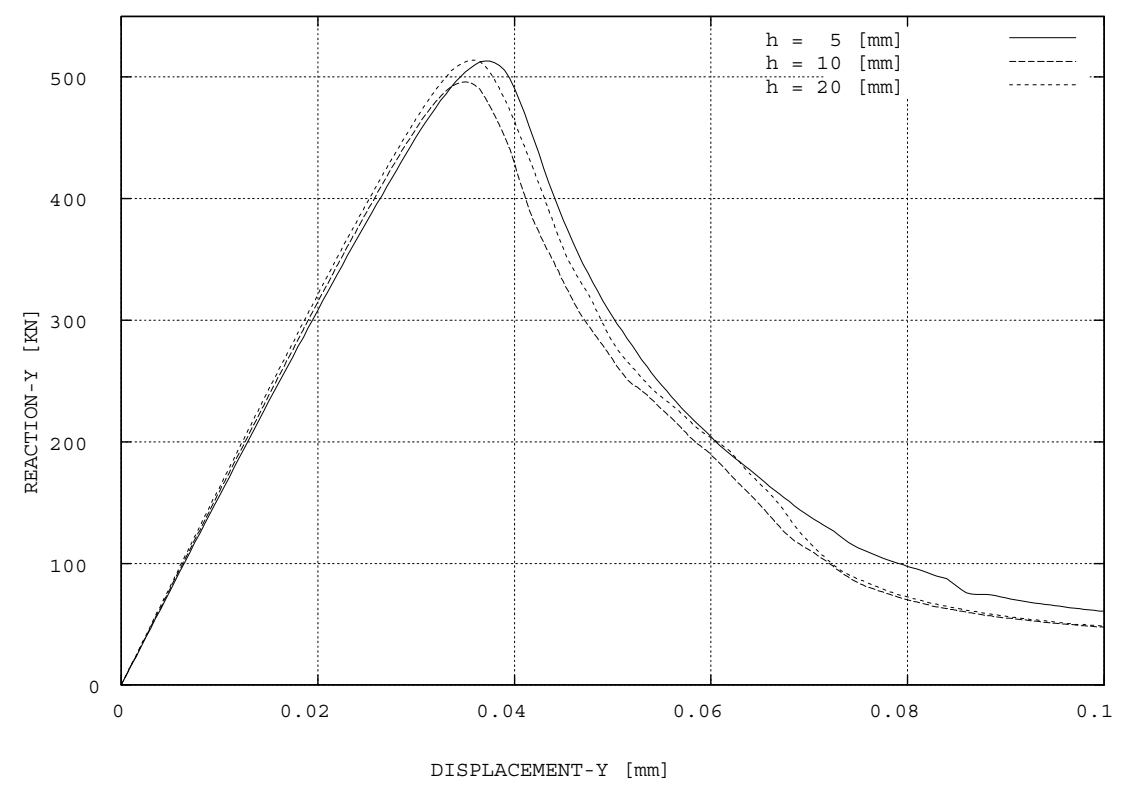

Figure 10: Load versus displacement for four point bending beam. Comparison between different mesh sizes

one crack, to $\mathcal{D}^{\text {tot }}=\mathcal{G}_{f} \times l_{\text {cr }} \times t=100 \times 0.25 \times 1=25 \mathrm{~J}$, where $l_{\text {cr }}$ is the length of the crack (aprox. $25 \mathrm{~cm}$ ) and $t$ is the thickness $(1 \mathrm{~m})$. The area under the curves is almost exactly equal to this value. No spurious brittleness is observed when the size of the elements is reduced.

Figure 11 shows the results obtained using the proposed formulation on the fine mesh. The three columns represent, respectively, the evolution, at four different time steps of the analysis, of: (a) the contours of vertical displacements, (b) the contours of maximum principal strain and (c) the maximum principal strain vectors. As in the previous example, the bottom figures show how, when the failure mechanism is fully developed, all the deformation concentrates in the formed cracks, while the elements outside these bands are mostly undeformed. Again, the resolution of the cracks is optimal for the mesh used. In the third column, it can be observed that the correct failure mechanism has been predicted although the directions of the computed maximum principal strain vectors (as the related vectors of maximum principal effective stress) are clearly dependent on the mesh bias, as they are not orthogonal to the crack path everywhere. Note in the left 


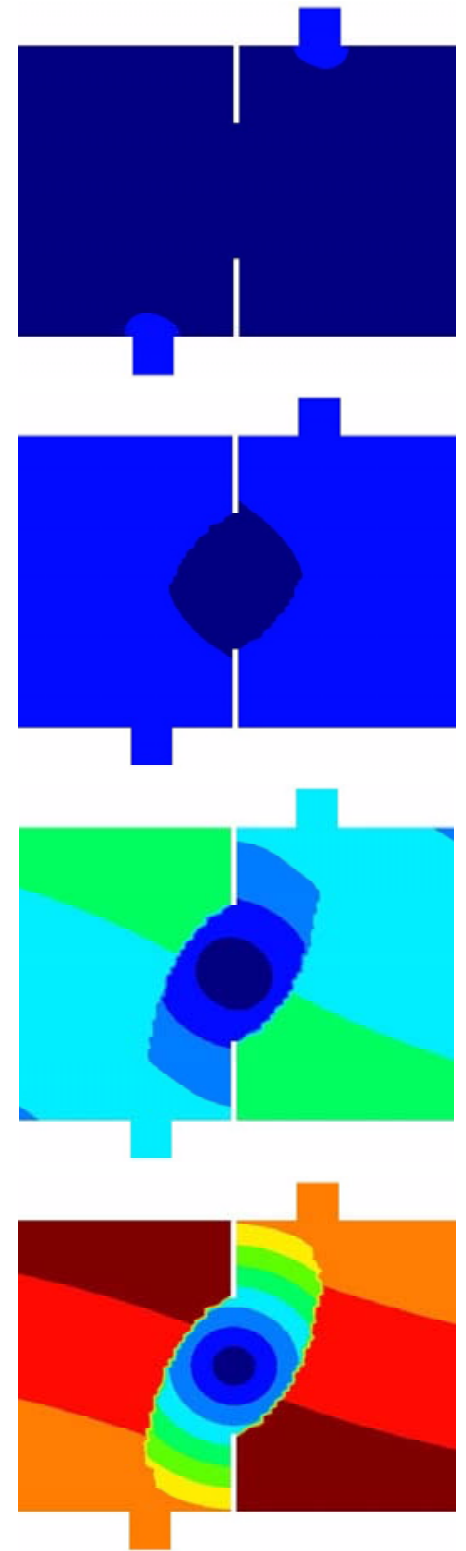

(a)
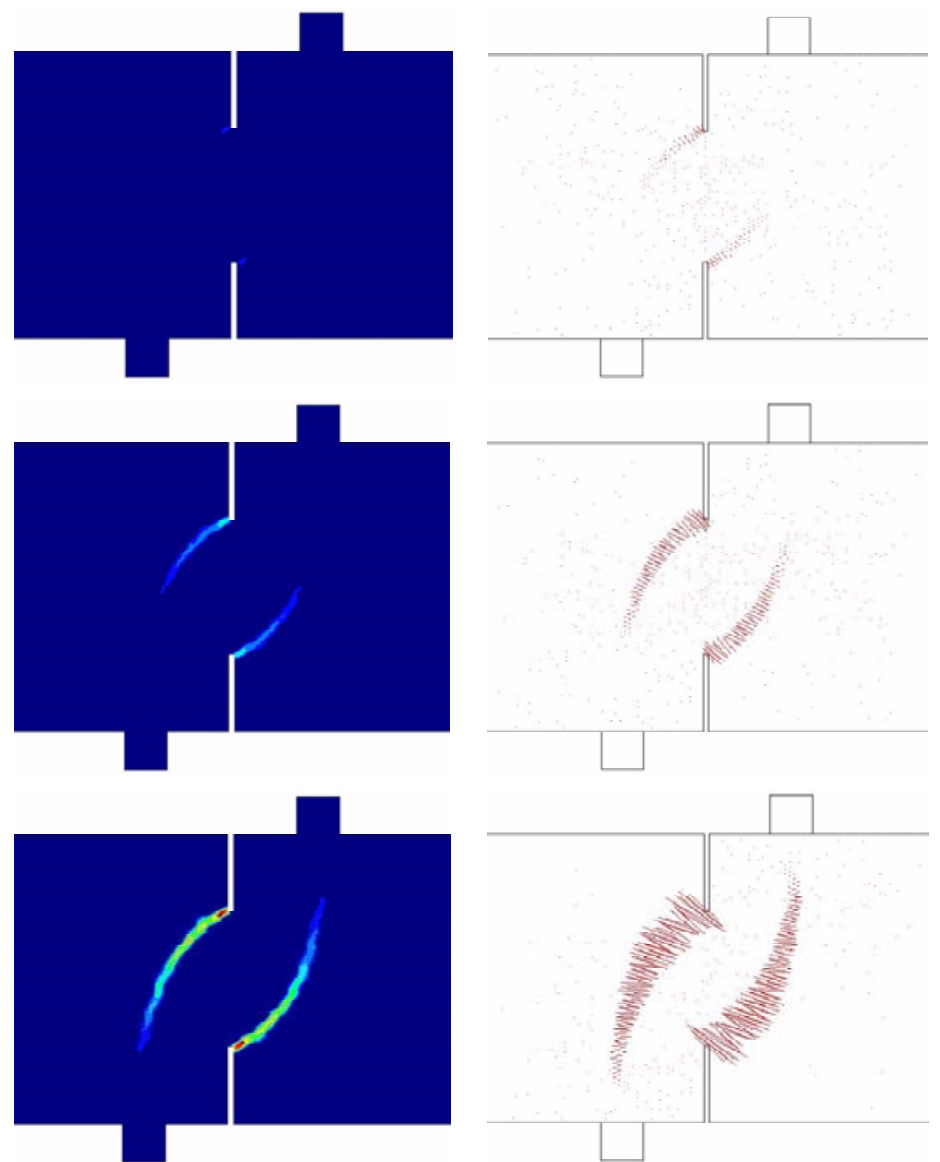

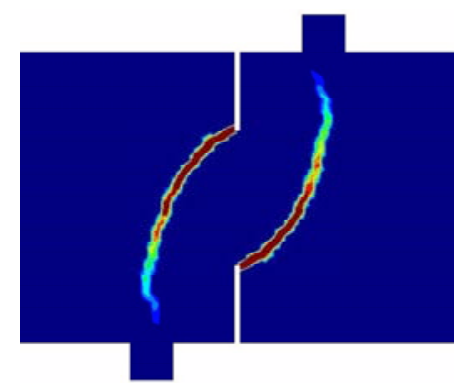

(b)

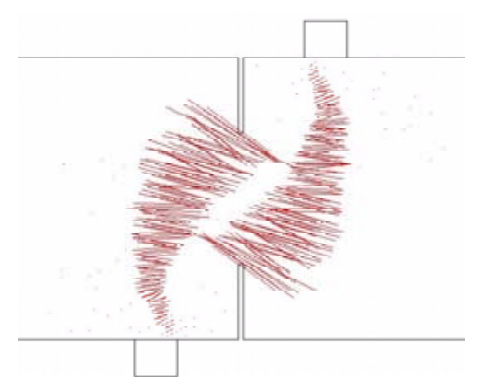

(c)

Figure 11: Results for four point bending beam using the proposed formulation. Evolution of: (a) vertical displacement, (b) maximum principal strain, (c) vectors of max. principal strain 
bottom plot how, once both cracks are formed, the central part of the beam rotates almost as a rigid body around the center of the beam.

For the coarser meshes, similar results are obtained, although the strain localization is smeared across a row of larger elements (see Fig. 9).

Finally, Figures 12a and 12b show the evolution, at three different time steps of the analysis, of: (a) the vertical displacements, (b) the maximum principal strain, along an horizontal line along the longitudinal axis of the beam which crosses both cracks. Again, it can be observed how displacements progressively localize into two very sharp jumps across one single element.

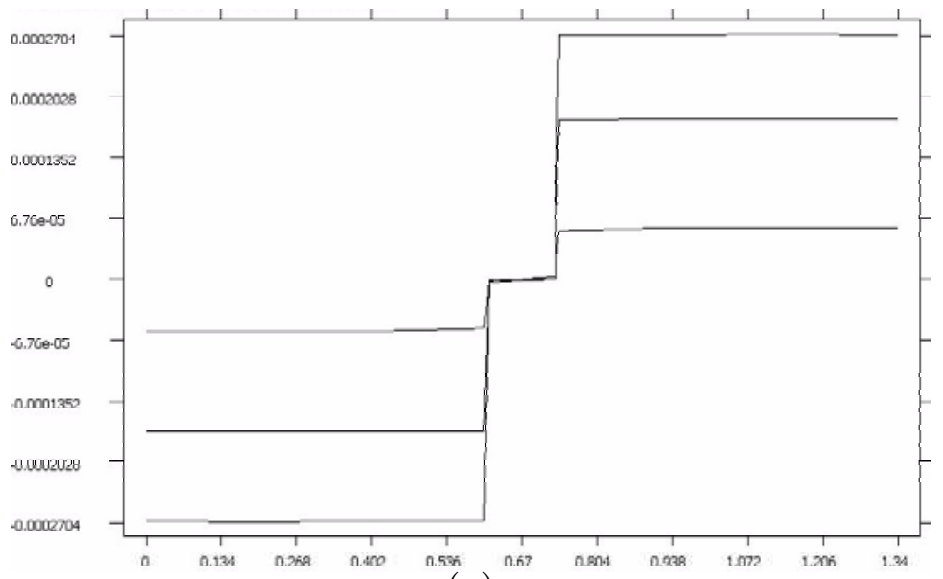

(a)

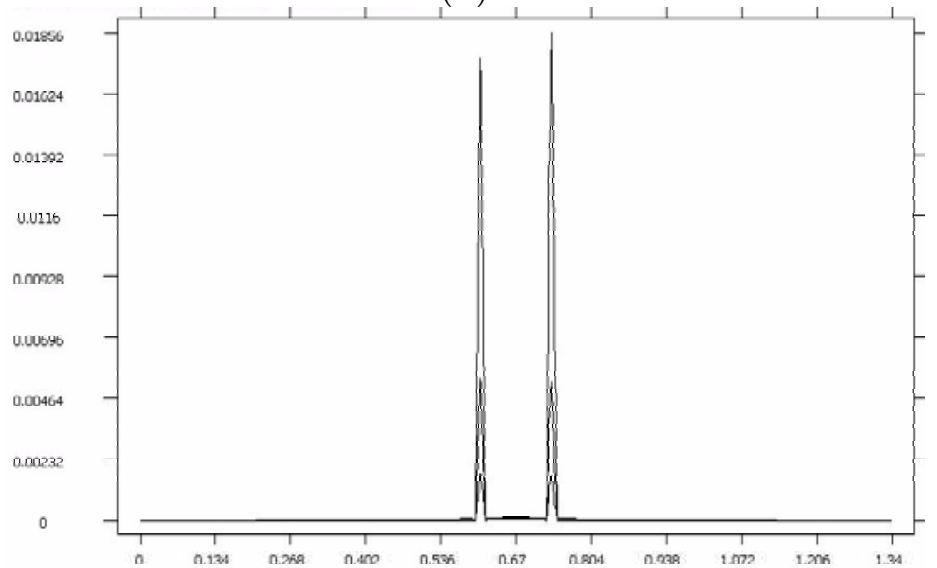

(b)

Figure 12: Evolution of the profiles along a vertical line of: (a) vertical displacement and (b) maximum principal strains for four point bending beam 


\section{Conclusions}

This paper shows that it is possible to tackle the solution of problems involving strain localization due to tensile straining (cracking) via the smeared crack approach, that is, using standard finite elements, such as linear triangles, and standard local constitutive models, such as an isotropic continuum damage model, and to obtain mesh objective results, such that: (a) the solution of the corresponding BVP can be computed in a step-by-step incremental manner, (b) the position and orientation of the localization paths (cracks) is independent of the directional bias of the FE mesh, and (c) the global post-peak load-deflection curves are independent of the size of the elements used.

This is attained by considering the determination of the direction of propagation of the strain localization band as a separate problem, coupled to that of solving the balance of momentum equation. The convenience of doing this is deduced from the stability analysis of the weak form of the associated discrete mechanical problem. Also, it stems from established practice with the discrete crack approach, both in the fracture and continuum mechanics frameworks.

The resulting formulation is convergent upon mesh refinement, virtually free of the spurious size and bias mesh dependence usually found when directly applying the smeared crack concept to strain localization problems. The derived method yields a robust scheme, suitable for engineering applications in $2 \mathrm{D}$ and $3 \mathrm{D}$.

Numerical examples show, on one hand, that the use of a crack propagation algorithm notoriously helps to avoid the dependence of the predicted failure mechanisms on the mesh directional bias; on the other, that relating the softening parameter of the constitutive model to the fracture energy of the material and to the size of the finite elements in the localization band enables to control the dissipated energy during the localization (fracture) process, yielding a correct structural response in the softening regime. Finally, computed solutions indicate that, as expected, continuous displacement interpolations can reproduce very sharp gradients if the mesh resolution is fine enough. 


\section{Acknowledgments}

The authors gratefully acknowledge the always helpful suggestions contributed by Prof. R. Codina in so many occasions.

\section{References}

[1] Galilei, G. (1638). Discorsi e dimonstrazioni matematiche intorno a due nuove scienze. English translation: Two new sciences, The Macmillan Company (1933), New York.

[2] Griffith, A.A. (1921). The phenomenon of rupture and flow of solids. Phil. Trans. Roy. Soc. (London), 22, 163-198.

[3] Barenblatt, G.I. (1959). On equilibrium cracks formed during brittle fracture. Prikl. Mat. Mech., 23, 3, 622-636 .

[4] Dugdale, D.S. (1960). Yielding of steel seets containing slits. J. of the Mechanics and Physics of Solids, 8, 100-104.

[5] Ngo, D. and Scordelis, A.C. (1967). Finite element analysis of reinforced concrete beams.ACI Journal, 64(14), 152-163.

[6] Tong, P. and Pian, T.H.H. (1973). On the convergence of the finite element method for problems with singularily. Int. J. Solids struct., 9, 313-321.

[7] Owen, D.R.J. and Fawkes, A.J. (1983). Engineering Fracture Mechanics, Pineridge Press, Swansea.

[8] Belytschko, T. and Black, T. (1999). Elastic crack growth in finite elements with minimal remeshing. Comp. Meth. in Appl. Mech. and Eng., $45(5), 601-620$.

[9] Möes, N., Dolbow, J. and Belytschko, T. (1999). A finite element method for crack growth without remeshing. Int. J. Num. Meths. in Engng., 46, 131-150.

[10] Sukumar, N., Möes, N., Moran, B. and Belytschko, T. (2000). Extended finite element method for three-dimensional crack modelling. Int. J. Num. Meths. in Engng., 48, 1549-1570. 
[11] Karihaloo, B.L. and Xiao, Q.Z. (2003). Modelling of stationary and growing cracks in FE framework without remeshing: a state-or-the-art review. Computers and Structures, 81, 119-129.

[12] Rashid, Y. (1968). Analysis of prestrssed concrete pressure vessels. Nuclear Engineering and Design, 7, 334-344.

[13] Hillerborg, A., Modeer, M., and Peterson, P.E. (1976). Analysis of crack formation and crack growth in concrete by means of F. M. and finite elements. Cement and Concrete Research, 6, 773-782.

[14] Bazant, Z.P. and Oh, B.H. (1983). Crack band theory for fracture of concrete. Material and Structures, 16, 155-177.

[15] Zienkiewicz, O.C., Pastor, M. and Huang, M. (1995). Softening, localization and adaptive remeshing: capture of discontinuous solutions. Comp. Mech., 17, 98-106.

[16] Zienkiewicz O.C., Huang, M. and Pastor, M. (1995). Localization problems in plasticity using finite elements with adaptive remeshing. Int. J. Num. Methods in Geomechanics, 19, 127-148.

[17] Aifantis, E.C., 1984. On the microstructural origin of certain inelastic models. Transactions ASME Journal of Engineering Materials Technology, 106, 326-330.

[18] de Borst, R. (1991). Simulation of strain localization: a reppraisal of the Cosserat continuum. Engineering Computations, 8, 317-322.

[19] Schereyer, H. and Chen, Z. (1986). One dimensional softening with localization. Journal of Applied Mechanics, ASME, 53, 791-797.

[20] Bazant, Z. and Jirásek, M. (2002). Nonlocal Integral Formulations of Plasticity and Damage: Survey of Progress. J. of Engineering Mechanics, ASCE, 128, 1119-1149.

[21] Vardoulakis, I. and Aifantis, E.C. (1991). A gradient flow theory of plasticity for granular materials. Acta Mechanica, 87, 197-217.

[22] de Borst, R. and Mulhaus, H.B. (1992). Gradient-dependent plasticity: formulation and algorithm aspect. Int. J. Num. Meths. in Engng., 35, 521-539. 
[23] Jirásek, M. (1998). Nonlocal models for damage and fracture: comparison of approaches. Int. J. Solids and Structures, 35, 4133-4145.

[24] de Borst, R. (2001). Some recent issues in computational failure mechanics. Int. J. Num. Meths. in Eng., 52, 63-95.

[25] Simone, A.. (2003). Continuous-Discontinuous Modelling of Failure. Ph. D. Thesis, TU Delft, The Netherlands.

[26] Needelman, A., (1987). Material rate dependence and mesh sensitivity in localization problems. Comp. Meth. in Appl. Mech. and Eng., 67, 68-75.

[27] Simo, J.C., Oliver, J. and Armero, F. (1993). An analysis of strong discontinuities induced by strain-softening in rate-independent inelastic solids. Computational Mechanics, 12, 49-61.

[28] Oliver, J. (1995). Continuum modeling of strong discontinuities in solid mechanics using damage models. Computational Mechanics, 17, 277296.

[29] Oliver, J., Cervera, M. and Manzoli, O. (1999). Strong discontinuities and continuum plasticity models: the strong discontinuity approach. Int. J. of Plasticity, 15, 319-351.

[30] Oliver, J., Huespe, A.E. Samaniego, E. and Chaves, W.V.. (2004). Continuum approach to the numerical simulation of material failure in concrete. Int. J. for Num. and Anal. Meth. in Geomechanics.,28, 609-632.

[31] Oliver, J. and Huespe, A.E. (2004). Continuum approach to material failure in strong discontinuity settings. Comp. Meth. in Appl. Mech. and Eng., 193, 3195-3220.

[32] Mosler, J. and Meschke, G. (2004). Embedded crack vs. smeared crack models: a comparison of elementwise discontinuous crack path approaches with emphasis on mesh bias. Comp. Meth. in Appl. Mech. and Eng., 193, 3351-3375.

[33] Feist, C., Kerber, W., Lehar, H. and Hofstetter, G. (2004). A comparative study of numerical models for concrete cracking. Proceedings of European Congress on Computational Methods in Applied Sciences and 
Engineering, ECCOMAS 2004, Neittaanmäki, P., Rossi, T., Korotov, S., Oñate, E., Periaux, J. and Knörzer, D. (eds.), Jyväskylä, Finland.

[34] Cervera, M., Chiumenti, M. and Agelet de Saracibar, C. 2003. Softening, localization and stabilization: capture of discontinuous solutions in J2 plasticity. Int. J. for Num. and Anal. Meth. in Geomechanics, 28, 373393.

[35] Cervera, M., Chiumenti, M. and Agelet de Saracibar, C. 2003. Shear band localization via local $\mathrm{J}_{2}$ continuum damage mechanics. Comp. Meth. in Appl. Mech. and Eng., 193, 849-880.

[36] Lemaitre, J. and Chaboche, J. L. (1978). Aspects phénoménologiques de la rupture par endommagement. J. Méc. Appl., 2, 317-365.

[37] Cervera, M., Oliver, J., and Faria, R. (1995). Seismic evaluation of concrete dams via continuum damage models. Earth. Engng. Struc. Dyn., $24,1225-1245$.

[38] Faria, R., Oliver, J., and Cervera, M. (1998). A strain-based plastic viscous-damage model for massive concrete structures. Int. J. Solids and Structures, 35(14):1533-1558.

[39] Simó, J. C. and Ju, J. W. (1987). Strain- and stress-based continuum damage models - I. formulation. Int. J. Solids and Structures, 23, 821840.

[40] Ju, J. W. (1989). On energy-based coupled elastoplastic damage theories: Constitutive modeling and computational aspects. Int. J. Solids and Structures, 7, 803-833.

[41] Oliver, J. (2000). On the discrete constitutive models induced by strong discontinuity kinematics and continuum constitutive equations. Int. J. Solids and Structures, 37, 7207-7229.

[42] Rots, J.G., P. Nauta, G.M.A. Kusters and J. Blaauwendraad (1985). Smeared Crack Approach and Fracture Localization in Concrete. Heron 30 (1).

[43] Rots, J.G. and J. Blaauwendraad (1989). Crack Models for Concrete: Discrete or Smeared? Fixed, Multi-Directional or Rotating? Heron 34 (1). 
[44] Oliver, J. (1989). A consistent characteristic length for smeared cracking models. Int. J. Num. Meth. Engng., 28, 461-474.

[45] Cervera, M., Oliver, J., and Manzoli, O. (1996). A rate-dependent isotropic damage model for the seismic evaluation of concrete dams. Earth. Engng. Struc. Dyn., 25, 987-1010.

[46] Faria, R., Oliver, J., and Cervera, M. (1999). On isotropic scalar damage models for numerical analysis of concrete structures. Research Publication, CIMNE, PI-170.

[47] Faria, R., Oliver, J., and Cervera, M. (2004). Modeling Material Failure in Concrete Structures under Cyclic Actions. J. of Structural Engineering, ASCE, 130, 1997-2005.

[48] Finchant, S, La Borderie, C. and Pijaudier-Cabot, G. (1999). Isotropic and anisotropic descriptions of damage in concrete structures. Mechanics of Cohesive-Frictional Materials, 4, 339-359.

[49] Kachanov, L. M. (1958). Time of rupture process under creep conditions. Izvestia Akademii Nauk, Old Tech Nauk, 8, 26-31.

[50] Dumstorff, P. and Meschke, G. (2004). Investigation of crack growth criteria in the context of the extended finite element method. Proceedings of European Congress on Computational Methods in Applied Sciences and Engineering, ECCOMAS 2004, Neittaanmäki, P., Rossi, T., Korotov, S., Oñate, E., Periaux, J. and Knörzer, D. (eds.), Jyväskylä, Finland.

[51] Grassl, P. and Jirásek, M. (2004). On mesh bias of local damage models for concrete: In Fracture Mechanics of Concrete Structures, Proceedings of 5th Int. Conference on Fracture Mechanics of Concrete and Concrete Structures, FraMCoS-5, V. C. Li et al. (eds.), Vail, Colorado, U.S.A.

[52] Cervera, M., Agelet de Saracibar, C. and Chiumenti, M. (2002). COMET: COupled MEchanical and Thermal analysis. Data Input Manual, Version 5.0, Technical report IT-308, www.cimne.upc.es.

[53] GiD: the personal pre and post-processor (2002). www.gid.cimne.upc.es. 